\title{
ABSTRACTS \\ SA HEART CONGRESS 2012
}

\section{Pump dysfunction in hypertensive hypertrophy: Impact of severity of blood pressure elevation in patients of African ancestry}

\author{
Haroon-ur-Rasheed Abbasi", Elena Libhaber*, Gavin Norton", Angela Woodiwiss" and Mohammed R. Essop" \\ "Division of Cardiology, Chris Hani Baragwanath Hospital, University of the Witwatersrand, Johannesburg, South Africa \\ \#Cardiovascular Pathophysiology and Genomics Research Unit, School of Physiology, Faculty of Health Sciences, \\ University of the Witwatersrand, Johannesburg, South Africa
}

Background: Hypertensive heart failure in developing communities may be associated with reduced pump function. Whether severity of hypertension impacts differently in patients with hypertensive hypertrophy with systolic dysfunction as compared to patients with hypertensive hypertrophy with preserved systolic function is not clear. We therefore evaluated the relative impact of blood pressure in patients with left ventricular hypertrophy $(\mathrm{LVH})$ with preserved left ventricular systolic function versus patients with LVH with reduced systolic function.

Methods: A single-centre study of I 75 Black African patients ( $55 \%$ female, age $=53.1 \pm 10.1$ years) with moderate to severe hypertension ( $\mathrm{HT}$ ) was performed. Using echocardiography, patients were grouped as follows: (I) HT with preserved left ventricular systolic function; and (2) HT with reduced left ventricular systolic function. All measurements were made using standard American Society of Echocardiography definitions. Low ejection fraction (EF) was defined as $<50 \%$ using the Simpsons method, LVH as LV mass index $>120 \mathrm{~g} / \mathrm{m}^{2}$ in $\mathrm{males}$ and $>100 \mathrm{~g} / \mathrm{m}^{2}$ in females. HT was defined as systolic or diastolic daytime ambulatory BP (ABPM) > $140 \mathrm{mmHg}$ or $95 \mathrm{mmHg}$ respectively. Twenty eight healthy normotensive subjects served as controls.

Results: There were no observed differences in any measure of blood pressure between patients with reduced EF ( 31 patients, I8\%) and preserved EF ( 144 patients, 82\%). The low EF group had significantly more male patients (81\% vs 37\%) but similar BMI (32.2 vs 30.7 ).

Conclusion: Blood pressure load and severity are similar in hypertensive patients with and without left ventricular dysfunction. These results would implicate factors other than blood pressure as important determinants of left ventricular remodelling in hypertension.

\section{Clinical and echocardiographic follow-up of the isolated left ventricular non-compaction (ILVNC) cohort at Chris Hani Baragwanath Hospital}

\section{Francois Botha*, Ferande Peters" and Mohammed R. Essop*}

"Division of Cardiology, Chris Hani Baragwanath Hospital, University of the Witwatersrand, Johannesburg, South Africa

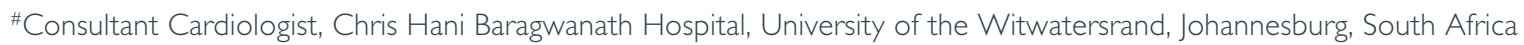

Background: Isolated left ventricular non-compaction (ILVNC) is a rare, genetic cardiomyopathy characterised by hypertribeculation of the left ventricle due to failure of normal embryonic compaction of the myocardium. Left ventricular systolic dysfunction, ventricular thrombus formation with cardiac thrombo-embolism and arrhythmias are frequently encountered. No data is available regarding follow-up of patients with ILVNC in Sub-Saharan Africa.

Aim: To describe the characteristics and outcomes of patients diagnosed with ILVNC attending the cardiomyopathy clinic at Chris Hani Baragwanath Hospital.

Methods: A prospective study was undertaken evaluating 4 I patients at the cardiomyopathy clinic at Chris Hani Baragwanath Hospital during January 2010 and March 2012. Clinical assessment, a screening echocardiogram for ventricular clots at four monthly intervals and formal research echocardiogram was performed on each patient annually.

Results: Mean follow-up was 12.8 +/- 5.0 months. The mean age was $39+/$ - 10.7 years at diagnosis. 13 male and 28 female patients. Mean left ventricular end diastolic diameter $56.8+/-8.6 \mathrm{~mm}$ and mean ejection fraction $32.2+/-12.1 \%$. The major complications at baseline were heart failure, 67\% were in NYHA class II/III, left ventricular thrombus formation in II(26\%). At follow-up 31.7\% were in NYHA II/III heart failure, 5 ( $13.1 \%)$ patients reported worsening heart failure of which 2 (4.8\%) required admission and none had left ventricular thrombi. Warfarin was 
prescribed in 29\% of patients with no bleeding complications. There were 5 deaths: I cardiac failure related and 4 sudden cardiac deaths. Conclusion: At one year follow-up, heart failure and sudden cardiac death remained a significant complication of ILVNC despite standard heart failure treatment and meticulous follow-up.

\section{First Melody ${ }^{\circledR}$ valve implantation in Africa}

\section{D.G. Buys and S.C. Brown}

Department of Paediatric Cardiology, University of the Free State, Bloemfontein, South Africa

Introduction: Congenital heart lesions with abnormalities involving the right ventricular outflow tract will require early surgical repair. Reintervention rates in these patients are very high due to outflow tract degeneration. The first percutaneous pulmonary valve was implanted by Professor Philipp Bonhoeffer in 2000. This valve was modified to develop the Melody ${ }^{\circledR}$ percutaneous pulmonary valve.

Methods: We describe the first 2 Melody ${ }^{\circledR}$ valve implantations in Africa. This was done in 2 male patients where the primary indication for valve implantation was increased right ventricular pressure and pulmonary insufficiency.

Results: Melody ${ }^{\circledR}$ valve implantation was successful in both patients with reduction of right ventricular outflow tract gradients. Both patients had no regurgitation post valve implantation.

Conclusion: Percutaneous pulmonary valves are safe and effective therapy for right ventricular outflow tract revalvulation. The Melody ${ }^{\circledR}$ valve can be implanted with a high success and low morbidity and mortality rates. Conduit lifespan can be extended and re-operation rates can be reduced.

\section{Untreated aortic stenosis (AS): guidelines for a tertiary setting}

\section{Malcolm Dalrymple-Hay, Ian Cox, Sam Melhuish, Tania Riches and Clinton Lloyd}

Derriford Hospital, Plymouth, Devon, United Kingdom

Introduction: We aimed to document the number of untreated patients with severe AS in a tertiary cardiac surgical centre and to establish the reasons why a valve intervention was not performed.

Methods: The echocardiography database for one calendar year, 2009 (4 086 studies) was interrogated and all patients with AHA criteria of severe AS were analysed.

Results: 530 patients had aortic stenosis, of these 175 were classified as severe. 79 (45\%) underwent treatment (75 conventional AVR, $4 \mathrm{TAVI}$ ); of the remainder 4 were lost to follow-up; and 92 (53\%) did not have a valve intervention. In the untreated group there were 34 males and the mean age was 82 years. A cardiologist was involved in 86 of those not treated; 10 were referred for surgery; and 76 were not referred. Of the 10 patients referred 2 died on waiting list; 2 awaited interventions; and 6 declined therapies. Of the 76 patients not referred in 26 it was decided that the AS was not severe despite only I having an aortic valve area $>1.0$. Of those patients not referred 9 had moderate LV function; 12 Dyspnoea; 2 Chest pain; and 3 Pre-syncope. The reasons patients were not referred included patient decision (14); reported asymptomatic status (15); symptoms not thought to be due to AS (5); advanced age (30); significant co-morbidity (34); limited life expectancy (25); high operative risk (29); and others (33). Review by the aortic stenosis multi-disciplinary team (MDT) supported no intervention in only 38 (46\%), but review of the remaining 44 patients.

Conclusion: In a tertiary setting a retrospective review suggested a significant number of patients with severe aortic stenosis were not offered any valve intervention. We believe to optimise care patients with an echocardiographic diagnosis of severe AS should all be reviewed by an aortic valve MDT. 


\section{Anomalous origin of the right pulmonary artery from the aorta: case reports and discussion}

\section{H. Dama}

Paediatric Cardiology Fellow, Department of Paediatrics, Nelson R. Mandela School of Medicine, University of KwaZulu-Natal, Durban, South Africa

Four cases of anomalous origin of the right pulmonary artery from the ascending aorta (AORPA) were seen at our hospital over the last 2 years:

Patient I presented at 6 years of age with cardiac failure. Echocardiography showed AORPA, a large PDA and features of pulmonary hypertension. Cardiac catheterisation demonstrated established pulmonary vascular obstructive disease which pre-cluded surgical correction. He was commenced on Sidenafil and now displays signs of clinical improvement.

Patient 2 presented at 26 days of age with apnoea. Echocardiography showed AORPA, a hypoplastic aortic arch, discrete distal coarctation and a large PDA. Surgical correction was performed at I month of age. Cardiac catheterisation and balloon dilatation of a re-coarcted segment was performed at 9 months of age. He is likely to require repeat intervention in the future.

Patient 3 presented at 23 days of age with cardiac failure. Echocardiography showed AORPA and a PDA. He required prolonged ventilation pre-op for sepsis and nosocomial pneumonia. Surgical correction was performed at 3 months of age. He was maintained on Sidenafil post-op and was clinically well at last follow-up.

Patient 4 presented at 6 days of age with shock. The diagnosis of AORPA and tubular hypoplasia of the aortic arch was made on echocardiography and confirmed by CT angiography. Clinical course was complicated by persistent nosocomial sepsis and hypotension with his condition deemed unsuitable for surgical correction. Intensive care was finally withdrawn after consultation with the family.

Anomalous origin of a pulmonary artery from the aorta as a major cardiac lesion is rare. Most patients develop early signs of cardiac failure. Echocardiographic diagnosis may be challenging. The natural history includes early death and the development of progressive pulmonary vascular disease in a significant number of patients. Associated aortic arch abnormalities may worsen the prognosis. Early diagnosis and surgical intervention are important in managing these patients.

\section{Protean manifestations of cardiac hydatidosis}

\section{Riaz Suleman Dawood", Ahmed R. Essop*, Robert W. Girdwood", Martin J. Sussman" and Mohammed R. Essop*}

"Chris Hani Baragwanath Academic Hospital, Milpark Hospital, University of the Witwatersrand, Johannesburg, South Africa \#Milpark Hospital, Johannesburg, South Africa

Introduction: Hydatid disease is a zoonosis caused by the cestode Echinococcus granulosus. It is endemic in certain parts of Sub-Saharan Africa. Humans are "accidental" intermediate hosts. Cardiac involvement is rare and not formally described in Southern Africa. Herein we describe the clinical, electrocardiographic, echocardiographic and serologic features of the disease and also describe surgical and pharmacologic management of four patients presenting with cardiac hydatidosis.

Methods: Retrospective review of case records identified 4 patients with histological confirmation of hydatid disease of the heart from which data was abstracted.

Results: All patients were native to Botswana and at some stage resided in rural parts of the country. Three were female and I male. Mean age was 29 years. Two patients presented with rhythm disturbances, the third with chest pain and the fourth was detected incidentally. Electrocardiography confirmed complete heart block in I patient and revealed marked repolarisation abnormality in another. Single, well circumscribed cystic lesions were seen in all patients on echocardiography. One patient had concomitant hydatid disease of the liver as well as features suggestive of detachment of the endocyst from the pericyst ("water lily" sign). Another had features suggestive of daughter cysts being present. Two patients had positive serology for Echinococcus granulosus of which I also had eosinophilia. All patients underwent cardiopulmonary bypass, and cysts were aspirated, with subsequent injection of hypertonic saline and reaspirated followed by excision. One patient required implantation of a permanent pacemaker at the time of surgical intervention due to destruction of the conducting system by the disease process, 
and another required mitral valve replacement subsequently due to severe mitral regurgitation. No hypersensitivity reaction was documented. All patients survived to I year follow-up. Histology revealed scolices in 2 patients and hooklets in the other patients which are pathognomonic for hydatid disease. All patients were treated with albendazole, I patient had praziquantel added.

Conclusion: Hydatid disease of the heart is unusual. Presentation is highly variable and requires a high index of suspicion. Echocardiography is the most useful investigation. Where appropriate, a combined surgical and pharmacologic approach offers the best clinical outcome.

\title{
Defensive coping and subclinical vascular disease risk - associations with autonomic exhaustion in Africans and Caucasians: the sympathetic activity and ambulatory blood pressure in Africans (SABPA) study
}

\author{
Andrea de Kock", Leoné Malan*, Mark Hamer" and NicoT. Malan* \\ "Hypertension in Africa Research Team (HART), School for Physiology, Nutrition and Consumer Sciences, North-West University, \\ Potchefstroom Campus, Johannesburg \\ \#Psychobiology Group, Department of Epidemiology and Public Health, University College London, London, United Kingdom
}

Objective: The defensive active coping response is a recognised cardiovascular risk factor in Africans, especially in men. It is uncertain whether autonomic dysfunction might be the underlying cause. We therefore investigated associations between salivary MHPG (3-methoxy-4hydroxyphenolglycol), as a marker of sympathetic activity, and subclinical vascular disease risk in defensive coping Africans and Caucasians.

Methods: The Coping Strategy Indicator questionnaire identified participants who preferably utilise defensive coping. Ambulatory blood pressure was monitored for 24 hours and carotid intima-media thickness (CIMT) was determined from ultrasound images, as an indicator of subclinical vascular disease risk. Salivary MHPG was analysed with high performance liquid chromatography.

Results: Defensive active coping Africans $(n=143$ ) showed overall poorer health than Caucasians ( $n=\mid 48)$, together with higher self-reported stress, alcohol abuse, hypertension, abdominal obesity, and risk of diabetes $(p \leq 0.05)$. African women demonstrated lower levels of MHPG compared with Caucasian women, although no differences in men were found. Furthermore, Africans revealed a trend of increased low grade inflammation and glycated haemoglobin which was associated with increased CIMT. There was an inverse association between MHPG and CIMT in African men $(n=30)$ with a high risk of subclinical vascular disease $[\beta=-0.22(-0.40,-0.03)]$.

Conclusion: Defensive active coping Africans are more at risk of subclinical vascular disease. When defensive coping fails, sympathetic hyperactivity may be followed by autonomic exhaustion and sympatho-adrenal-medullary system desensitisation, resulting in pathology.

\section{Glucose and insulin: emerging metabolic therapy against acute heart failure (AHF)}

\section{Gaurang Deshpande, Sandrine Lecour and Lionel H. Opie}

Department of Medicine, University of Cape Town, Observatory, South Africa

Purpose: Using an isolated rat heart model to study AHF and recovery, in hearts subject to hypotension, hyperadrenergic stimulus, elevated fatty acids and reduced glucose, we aimed to explore the effect of metabolic modulators i.e. high glucose and insulin on functional recovery. We hypothesize that glycolysis protects against these adverse effects.

Methods: AHF was induced by reducing perfusion pressure to $20 \mathrm{~cm} \mathrm{H}_{2} \mathrm{O}$ and increasing free fatty acids (FFA) to $1.3 \mathrm{mM}$ in presence of adrenaline $\left(10^{-8} \mathrm{M}\right)$ while reducing glucose to $2.5 \mathrm{mM}$. Recovery phase was achieved by a perfusion pressure of $100 \mathrm{~cm} \mathrm{H}_{2} \mathrm{O}$ with unchanged FFA levels ( $1.3 \mathrm{mM})$. High glucose $(\mathrm{HG})(1 \mathrm{l} .1 \mathrm{mM})$ was tested in both AHF and recovery phase. Insulin was added only in the recovery phase. Results: In the AHF phase, substrate changes with added adrenaline and hypotension reduced heart rate (HR), left ventricular developed pressure (LVDP) and increased diastolic pressure. HG reduced the abnormal diastolic pressure (6.5I \pm 0.9 vs $54.5 \pm 13.0 \mathrm{mmHg}$; $<<0.0$ I) thus increasing relaxation.

Recovery phase: In the recovery phase, with no added adrenaline but high FFA in the perfusate, added insulin increased HR (I $68.5 \pm 34.5$ BPM (beats per minute) vs $36.7 \pm 25.0$ BPM; $p<0.01$ ). LVDP or rate pressure products (RPP) were unchanged. HG throughout both phases 
improved recovery versus low glucose (LG) $(2.5 \mathrm{mM})$. HR recovered (229.6 \pm 25.6 vs $36.7 \pm 25.0$ BPM; p<0.00 I), RPP was almost 4.3 times better in high glucose (in AHF) hearts $(\mathrm{p}<0.00 \mathrm{I})$.

Conclusion: In our model of AHF in the acute phase, HG decreases diastolic pressure. In the recovery phase, both high glucose and insulin improve the functional recovery by increasing HR and reducing the diastolic pressure. Thus, glycolysis protects from diastolic dysfunction in both phases of AHF.

\section{Williams-Beuren syndrome and Ebstein's anomaly variant: a rare association}

\section{Adele Dippenaar, Lungile Pepeta and Samina Mahmud Yakoob}

Division of Paediatric Cardiology, Dora Nginza Hospital, Port Elizabeth Hospital Complex, Walter Sisulu University, Port Elizabeth, South Africa

Background: Williams-Beuren syndrome (WBS), is rare (1:10 000 to 1:50 000) and is caused by a hemizygous contiguous gene deletion on chromosome 7q I 1.23 encompassing the elastic gene (ELN) and 25-35 other genes, all of which is detectable by fluorescent in situ hybridisation (FISH). It is characterised by multiple anomalies including typical facial dysmorphism, congenital heart defects, growth deficiency, mental retardation, a-typical behavioural profile and occasionally idiopathic infantile hypercalcaemia. Cardiovascular disease presents as elastin arteriopathy which is generalised, commonly presenting as supra-valvular Aortic Stenosis (AS) or supra-valvular Pulmonary Stenosis (PS). The association of this syndrome with Ebstein's anomaly is very rare. We present our case.

Case presentation: A I5-year-old boy presented as a referral from the adult cardiology services with dysmorphic features, mental challenge and suspected acyanotic congenital heart disease for a second opinion. He had been seen as an infant and was lost to follow-up. On examination he had the phenotypic features associated with WBS which was subsequently confirmed by FISH. Echocardiogram revealed the presence of an Ebsteinoid tricuspid valve with Right Ventricular Non-compaction and severe tricuspid regurgitation. The patient was commenced on diuretics and underwent successful surgical repair of his tricuspid valve. He developed transient complete heart block postoperatively. All anti-failure medication was stopped on follow-up. He remains with mild residual tricuspid valve regurgitation and prolapse of the anterior tricuspid valve leaflet.

Conclusion: Congenital intra-cardiac anomalies associated with William-Beuren syndrome are extremely rare. To our knowledge, only I case of William-Beuren syndrome associated with Ebstein's anomaly has been reported at our hospital.

\section{Sutureless aortic valve replacement: catheter-based transapical versus direct transaortic implantation}

\section{Mirko Doss}

Department of Thoracic and Cardiovascular Surgery, J.W. Goethe-University, Frankfurt, Germany

Background: Catheter-based aortic valve implantation leaves the calcified native leaflets in the aortic root. Upon valve deployment, they are pushed to the side, potentially compromising coronary perfusion, exerting pressure on the conduction system and limiting maximum effective orifice area. In this study, we evaluated peri-operative outcomes of an alternative self-expanding sutureless valve that is inserted via a conventional aortotomy, allowing for complete debridement of calcified native aortic leaflets and annulus.

Methods: A total of 80 patients were included in the study, all requiring bioprosthetic valve replacement for aortic valve stenosis. In the first group 29 patients had isolated catheter-based transapical aortic valve implantation using the Sapien Edwards prosthesis. The second group of patients $(n=80)$ had direct transaortic valve replacement, using the self-expandable ATS 3F Enable prosthesis. Implantation of the latter device utilized cardio-pulmonary bypass, thus debridement of the calcified native leaflets was possible in all cases. In hospital clinical outcomes, echocardiography findings and routine blood laboratory parameters were evaluated.

Results: Thirty day mortality in the transapical group was 17\% versus 11\% in the transaortic group. There were 2 conversions in each group. There was I valve migration, I complete heart block and 2 cases of coronary obstruction in the transapical group. Implantation time was 
8 minutes in the transapical group versus 10 minutes in the transaortic group. There were 4 paravalvular leaks in the transapical group and none in the transaortic group.

Conclusion: Aortic valve replacement can be performed safely with both sutureless devices. The transaortic approach adds the advantage of allowing for resection of native leaflets and performance of concomitant procedures. Laboratory findings showed elevated creatine kinase $\mathrm{MB}$ fraction in the transapical group and comparable inflammatory parameters, despite the use of cardio-pulmonary bypass in the transaortic group.

\section{Review of truncus arteriosus cases seen at Chris Hani Baragwanath Academic Hospital: a "vanishing syndrome"}

\section{G. Dumani, B. Fourie, P.A. Adams and A.M. Cilliers}

Division of Paediatric Cardiology, Department of Paediatrics and Child Health, Chris Hani Baragwanath Academic Hospital, University of the Witwatersrand, Johannesburg, South Africa

Introduction: Truncus arteriosus is an uncommon congenital heart disease characterised by a single arterial trunk with a single semilunar valve and supplying blood flow to the aorta, pulmonary arteries and coronary arteries. Associated high morbidity and mortality is secondary to pulmonary hypertension and associated complex cardiac lesions.

Methods: Retrospective clinical review of 45 cases between 1978 and 2012 presenting to the Division of Paediatric Cardiology at the Chris Hani Baragwanath Academic Hospital. Data related to clinical features, diagnosis, operative procedures, pre-operative and post-operative follow-up was collected.

Results: 45 patients ( 18 males and 27 females) were diagnosed. The most common presenting features were dyspnoea, tachypnoea, a cardiac murmur and congestive cardiac failure. Median age at presentation was 8 weeks. Diagnosis was made with echocardiography in all the patients. Patients were classified according to type of truncus arteriosus as follows: 31 had Type 1; 7 had Type 2; 1 had Type 3 and 6 patients were not classified. Median time to surgery after diagnosis was 8 weeks: 12 patients had successful surgery; 18 were lost to follow-up pre-operatively; and 4 were lost to follow-up post-operatively. 13 patients died pre-operatively while 4 patients died post-operatively.

Conclusion: Mortality is up to $80 \%$ in unoperated truncus arteriosus during the first year of life. Early diagnosis with good pre-operative care is essential to reduce morbidity and mortality. Morbidity and mortality is related to associate complex lesions. Excellent results are achieved with early repair and prevent irreversible pulmonary vascular disease.

\section{The road to a Fontan and beyond - often a bumpy one: a complicated case of tricuspid atresia with a lot of diagnostic and treatment dilemmas}

\section{B. Fourie, A.M. Cilliers, P.E. Adams and G.C. Dumani}

Division of Paediatric Cardiology, Department of Paediatrics and Child Health, Chris Hani Baragwanath Academic Hospital, University of the Witwatersrand, Johannesburg, South Africa

We present a 25-year-old male that was diagnosed at I month of age with Tricuspid atresia IB. This patient has had numerous cardiac catheterisations and surgeries. These include a classical left-sided Blalock Taussig shunt in 1987, a modified right-sided bi-directional Glenn shunt in 1995 and an internal/external Fontan in 2012. He has also undergone 7 cardiac catheterisations up to this date, 2 of which were interventional procedures. He had a balloon angioplasty of a Left Pulmonary Artery stenosis caused by the previous Blalock Taussig shunt in 2003, followed by a balloon angioplasty of the stenosed classic Blalock Taussig shunt and stenting of the Left Pulmonary Artery stenosis in 20 I0. The patient had severe polycythaemia before the Fontan surgery and also complained of right sided parasthesia and dizziness. During the Fontan surgery done in 2012 an organised clot was noted within the Left Pulmonary Artery stent with no blood flow distally. This obstruction could not be resolved intra-operatively. Further management regarding this left pulmonary artery occlusion remains a dilemma post-surgery. The patient currently has a one lung Fontan circulation and despite this he has improved post-operatively with an increase in systemic saturations and resolution of his severe polycythaemia. 


\title{
The effects of acute ethanolamine administration on isoprenaline-induced myocardial infarction in adult Wistar rats
}

\author{
Christie Garson, Asfree Gwanyanya, Kishor Bugarith and Roisin Kelly-Laubscher \\ Department of Human Biology, Faculty of Health Sciences, University of Cape Town, Observatory, South Africa
}

Introduction: Cardiovascular stress, such as that due to the synthetic catecholamine, isoprenaline (iso), can induce myocardial infarction (MI). Ethanolamine (etn) is a biogenic amine, found in certain food types, that protects the isolated rat heart against ischaemia-reperfusion injury. The aim of this study was to investigate the cardioprotective effects of acute etn on iso-induced MI.

Methods: Male Wistar rats (250-350g) were divided into four groups: Control (saline injections only), iso + etn (iso 67mg/kg, s.c. and etn $10 \mathrm{mg} / \mathrm{kg}$ i.p.), iso $(67 \mathrm{mg} / \mathrm{kg}$, s.c. and a saline injection), and etn (saline injection and $10 \mathrm{mg} / \mathrm{kg}$ i.p.). Rats were anaesthetised with sodium pentobarbitone $(60 \mathrm{mg} / \mathrm{kg}$, i.p.) and mechanically ventilated with room air. Arterial blood pressure was measured from the right carotid artery and the electrocardiogram (ECG) was recorded from surface leads. Infarct size was measured using triphenyltetrazolium chloride (TTC) staining and the byproducts of lipid peroxidation were quantified.

Results: Iso administration significantly increased the infarct size $(62.0 \pm 3.9 \%, \mathrm{p}<0.00 \mathrm{I})$ (mean $\pm \mathrm{SEM})$ compared to control rats that displayed a background TTC-negative artefact $(23.7 \pm 2.7 \%)$. The heart weight/body weight $(\mathrm{HW} / \mathrm{BW})$ ratio was also significantly raised in iso rats $(4.6 \pm 0.1, \mathrm{p}<0.00 \mathrm{I})$ compared to control rats $(3.4 \pm 0.02)$. Iso + etn $(5.0 \pm 0.2 \mathrm{p}<0.05)$ treated rats displayed significantly greater HW/BW ratio compared to iso-treated rats. However, there was no significant difference in infarct size between iso and iso + etn. Iso + etn treated rats had a lower death rate (1.9\%) compared to iso-treated rats (11.3\%). There was a significant increase in the amount of conjugated dienes $(C D)$ in iso rats $(59.56 \pm 2.6 \mu \mathrm{mol} / \mathrm{L})$ compared to control rats $(39.9 \pm 4.0 \mu \mathrm{mol} / \mathrm{L}, \mathrm{p}<0.05)$. There was no significant difference in the generation of $\mathrm{CD}$ s between control and iso + etn treated rats.

Conclusion: In summary, etn seems to protect the rat from iso-induced Ml; however the possibility of increased cardiac hypertrophy needs further investigation.

\section{The effects of magnesium administration on isoprenaline-induced acute myocardial infarction in adult Wistar rats}

\section{Christie Garson, Roisin Kelly-Laubscher, Kishor Bugarith and Asfree Gwanyanya}

Department of Human Biology, Faculty of Health Sciences, University of Cape Town, Observatory, South Africa

Introduction: Magnesium $\left(\mathrm{Mg}^{+}\right)$is widely used in the treatment of cardiac arrhythmias and hypertensive disorders, but its effects on ischaemia remain unclear. The aim of this study was to investigate the effects of acute $\mathrm{Mg}^{+}$administration on isoprenaline (iso)-induced acute myocardial infarction (MI).

Methods: Male Wistar rats (250 - 350g) were divided into four groups and treated as follows: Control (saline injections only), iso + Mg (iso $67 \mathrm{mg} / \mathrm{kg}$, s.c. + Mg $270 \mathrm{mg} / \mathrm{kg}$ i.p.), iso $(67 \mathrm{mg} / \mathrm{kg}$, s.c. and a saline injection), and $\mathrm{Mg}$ (saline injection and $270 \mathrm{mg} / \mathrm{kg}$ i.p.). Rats were anaesthetised 24 hours later with sodium pentobarbitone $(60 \mathrm{mg} / \mathrm{kg}$, i.p.), mechanically ventilated with room air and electrocardiogram was recorded. Left ventricular blood pressure was measured via the right carotid artery. Infarct size was measured using triphenyltetrazolium chloride (TTC) staining and quantified as a percentage of the total ventricular area.

Results: Iso induced an infarct of $70.4 \pm 4.0 \%$ (mean \pm SEM) compared to control rats that displayed a background TTC-negative artefact $(22.5 \pm 1.7 \% ; \mathrm{p}<0.00 \mathrm{I}) . \mathrm{Mg}^{+}$did not change the infarct size in iso-treated rats (61.2 $\left.\pm 3.4 \% ; \mathrm{p}>0.05\right)$. Iso decreased the rate of ventricular relaxation as indicated by $\mathrm{dP} / \mathrm{dt} \min (-5550 \pm 220 \mathrm{mmHg} / \mathrm{s})$ compared to controls $(-79 \mathrm{I} 4 \pm 502 \mathrm{mmHg} / \mathrm{s} ; \mathrm{P}<0.0 \mathrm{l})$, but did not change the heart rate. $\mathrm{Mg}^{+}$had no effect on $\mathrm{dP} / \mathrm{dt}$ min in iso-treated rats $(-5882 \pm 466 \mathrm{mmHg} / \mathrm{s} ; \mathrm{p}>0.05)$ or on heart rate. Iso decreased the amplitude of the R-wave $(0.18 \pm 0.03 \mathrm{mV}$, compared to controls $0.58 \pm 0.06 \mathrm{mV} ; \mathrm{p}<0.00 \mathrm{I})$, but had no effect on the S-wave. $\mathrm{Mg}^{+}$had no effect on $\mathrm{R}$ or $\mathrm{S}$-wave amplitudes on its own, but decreased the S-wave and not the R-wave in iso-treated rats (S-wave amplitude: $-0.06 \pm 0.02 \mathrm{mV}$ compared to controls $-0.3 \mathrm{I} \pm 0.08 \mathrm{mV} ; \mathrm{p}<0.05$ and to iso $-0.13 \pm 0.07 \mathrm{mV} ; \mathrm{p}<0.05) . \mathrm{Mg}^{+}$increased the loss of body weight in iso-treated rats $(-4.3 \pm 0.9 \%$ compared to iso $-3.8 \pm 0.8 \% ; p<0.0$ I and $0.6 \pm 0.5 \%$ for controls; $p<0.00$ I) but there were no differences in mortality. 
Conclusion: $\mathrm{Mg}^{+}$neither decreases nor increases the size of the iso-induced infarct. However recent studies suggest that body weight loss after $\mathrm{Ml}$ in rats can be used as a marker of early heart failure development. Therefore the increased weight loss induced by $\mathrm{Mg}^{+}$in this study, may indicate adverse effects during its use in cardiac disease treatment.

\title{
Overcoming the limitations of current echocardiographic criteria used in the diagnosis of subclinical rheumatic heart disease
}

\section{Philip G. Herbst, Zaheera Cassimjee, Alfonso J.K. Pecoraro and Anton F. Doubell}

Division of Cardiology, Department of Medicine, University of Stellenbosch and Tygerberg Hospital, South Africa

Purpose: Current echocardiographic criteria for the diagnosis of subclinical rheumatic heart disease (RHD) have limitations: The 200। World Health Organisation Criteria (WHOC) is based on differentiating pathological from physiological mitral regurgitation (MR). New diagnostic criteria were proposed by Marijon et al in 2009, the so-called Combined Criteria (CC), which in addition to the presence of MR includes morphological features of the rheumatic valve. However, these criteria do not address the most specific feature of the rheumatic process, namely commissural fusion. In this study we test the diagnostic ability of the WHOC and CC, and investigate the utility of adding a commissural assessment.

Methods: Patient selection was based on a keyword search of echocardiography reports and inclusion of the first 15 patients fulfilling criteria of each of our cohorts: Cohort I: Mitral stenosis (MS); Cohort 2: RHD as defined by CC but without MS; Cohort 3: Dilated cardiomyopathy with leaflet restriction due to mitral valve tethering; Cohort 4: Normal controls. Detailed transthoracic echocardiography was performed including a direct and indirect assessment of the mitral valve commissures. We tested the sensitivity and specificity of current WHOC and CC to detect RHD against our Commissural Fusion Criteria (CFC) and described and compared the mitral valve commissures in Cohorts I-4.

Results: The WHOC demonstrated poor sensitivity in selecting patients with RHD as evidenced by the presence of MS, and poor specificity in differentiating non-rheumatic MR. The CC fared better in terms of specificity. The CFC outperformed both the WHOC and the CC in sensitivity and specificity.

Conclusion: We demonstrate the limitations of current echocardiographic criteria in diagnosing RHD. Both the WHOC and CC are undermined by the inclusion of $M R$ as a criterion. They necessarily exclude all RHD patients without MR, the extreme example being pure MS patients and incorrectly include patients with non-pathological MR. The CC showed improved specificity compared to the WHOC by inclusion of valve morphology but the exclusion of commissural fusion, the most specific feature of the rheumatic process, weaken these criteria. We propose that excluding MR as a diagnostic criterion and including a commissural analysis, will strengthen current criteria.

\section{Cardioprotective and anti-hypertensive effects of Prosopus glandulosa in a rat model of pre-diabetes}

\author{
Barbara Huisamen*, Cindy George*, Daneel Dietrich" and Amanda Lochner* \\ "Division of Medical Physiology, Department of Biomedical Sciences, Faculty of Health Sciences, University of Stellenbosch, South Africa \\ \#Department of Biomedical Sciences, University of Western Cape, Bellville
}

Introduction: Obesity and type 2 diabetes present with 2 debilitating complications: hypertension and heart disease. The dried and ground pods of Prosopus glandulosa is currently marketed as a food supplement with anti-hypertensive properties in South Africa. We determined the efficacy of $\mathrm{P}$ glandulosa as an anti-hypertensive agent and its myocardial protective ability.

Methods: Male Wistar rats were rendered (a) pre-diabetic (diet-induced obesity: DIO) or (b) hypertensive (high fat diet: HFD). DIO animals were treated with $\mathrm{P}$ glandulosa ( $100 \mathrm{mg} / \mathrm{kg} / \mathrm{day}$, 8 weeks) and compared to age-matched controls. Hearts were perfused ex vivo to determine infarct size. Biometric parameters were determined at time of sacrifice. CIRKO mice were similarly treated with P glandulosa and infarct size determined. HFD animals were treated from the onset of the diet or from weeks 12-16, using Captopril (50mg/kg/day) as positive control. Blood pressure was monitored weekly. 
Results: DIO rats and CIRKO mice: P glandulosa ingestion significantly reduced infarct size after ischaemia/reperfusion. Proteins of the PI-3kinase/PKB/Akt survival pathway were affected in a manner supporting cardioprotection. HFD model: P glandulosa treatment both prevented and corrected the development of hypertension, also reflected in alleviation of water retention.

Conclusion: P glandulosa is cardioprotective and infarct sparing as well as anti-hypertensive without affecting the body weight or the intraperitoneal fat depots of the animals. Changes in the PI-3-Kinase/PKB/Akt pathway may be causal to protection. Results indicated water retention, possibly coupled to vasoconstriction, in the HFD animals while ingestion of P glandulosa alleviated both. Treatment of pre-diabetes, type 2 diabetes or hypertension with $\mathrm{P}$ glandulosa may have immense beneficial health effects.

\section{Racial variability in telomere length and risk of coronary artery disease}

\section{Sajidah Khan", Anil Chuturgoon", Devapragasen Moodley\# and Alisa Phulukdaree\#}

"Department of Cardiology, University of KwaZulu-Natal, Durban, South Africa

\#Department of Medical Biochemistry, University of KwaZulu-Natal, Durban, South Africa

Introduction: Shorter telomere length is consistently associated with increased cardiovascular risk and mortality and is thought to precede the development of atherosclerotic coronary artery disease (CAD). Subsets of our population are at different ends of the risk spectrum for CAD. The risk variance may be due to dissimilar gene pools. Telomere length $(T L)$ is not only determined by cell replication from the ageing process, but is also influenced by genetic inheritance and the cumulative effect of exposure to environmental risk factors.

Aim: Investigate the major determinants of $T L$ in a biracial cohort of healthy, young adults extracted from a population with a very high prevalence of CAD as well as a low prevalence population.

Method: The study design was cross-sectional with a population-based, random selection of normal, asymptomatic participants matched for age and gender. Mean TL was determined from leukocyte DNA through a modified quantitative polymerase chain reaction based-assay and reported as the relative telomere/single copy gene (T/S) ratio. Fasting blood samples were collected for lipid profile, insulin levels, systemic markers of inflammation and oxidative stress. All participants underwent an oral glucose tolerance test and were subjected to standard symptom-based questionnaires, clinical examination and exercise stress test.

Results: 100 Indian males and 87 Black males (85\% ethnic Zulus) with a mean age of 37 years participated. TL was significantly longer in the Black cohort with a T/S ratio of 0.89 versus 0.67 in Indians $(p<0.000 \mathrm{I})$. There were no significant differences in the number of current smokers, BMI, systolic and diastolic blood pressure, levels of HDL-cholesterol and hsCRP between the groups. However, there were significant differences in family history of premature CAD (32\% Indians) vs 0\% (Blacks), measures of abdominal obesity, lipid parameters other than HDL-cholesterol and prevalence of Type 2 diabetes and pre-diabetes between the two groups. On multi-variate analysis only race $(p<0.00 \mathrm{I})$ and pre-diabetes $(p=0.0 \mathrm{I})$ were independently associated with mean $T L$.

Conclusion: Telomere length was significantly longer in Black Africans compared to Indians in this cohort of healthy young adults, perhaps reflecting a genetic susceptibility amongst Indians, and may partly explain the considerable difference in the incidence of CAD between these population groups. 


\title{
Feline hypertrophic cardiomyopathy associated with the A3 I P mutation in MYBPC3, is caused by production of mutated protein $C$ with reduced affinity between the MyBP-C COCI domain and actin
}

\author{
Craig Kinnear", Mia Godiksen",Tina Nielsen†, Peter Højrup๋, Sara Granström\#, Inga A. Laursen", Jørgen Koch", \\ William J. McKenna ${ }^{\circ}$ and Michael Christiansen ${ }^{* *}$ \\ "Department of Biomedical Sciences, University of Stellenbosch, South Africa \\ \#Department of Small Animals Diseases, Faculty of Life Science, University of Copenhagen, Denmark \\ tInstitute of Biochemistry and Molecular Biology, University of Southern Denmark, Odense, Denmark \\ 抽epartment of Clinical Biochemistry and Immunology, Statens Serum Institute, Copenhagen, Denmark \\ 'The Heart Hospital, University College of London, United Kingdom \\ **:Department of Clinical Biochemistry and Immunology, Statens Serum Institute, Copenhagen, Denmark
}

Introduction: Hypertrophic cardiomyopathy $(\mathrm{HCM})$ is a primary disorder of the myocardium in both man and cat, with serious clinical manifestations such as stroke, heart failure and sudden cardiac death. Human and feline HCM (fHCM) are clinically comparable, thus HCM in Maine Coon (MC) cat is an animal model of human disease. In MC cats a large proportion ( 50\%) of the fHCM cases have been associated with the missense mutation, c.9|G>C, in the MYBPC3 gene coding for P.A3IP in cardiac myosin binding protein C (cMyBP-C). The mutation is located in the $\mathrm{NH}$ 2-terminal $\mathrm{COCI}$ domain of cMyBP-C which is believed to interact with actin in the sarcomere. The objective of this study was to investigate the pathogenic mechanism of the c.9 IG>C MYBPC3 mutation associated with feline HCM.

Method: Heart tissue was obtained from MC cats genotyped for the mutation and the presence of the mutated protein was examined by SDS-PAGE and mass spectrometry (MS). Yeast-2-hybrid $(\mathrm{Y} 2 \mathrm{H})$ analysis was used to assess the effect of the mutation on the interaction between $\mathrm{CMyBP-C}$ and actin. The publicly available NMR-based structure of the CO domain was used to calculate and visualise the consequences of substituting the proline for the alanine residue.

Results: Mass spectrometric analysis revealed expression of mutated CMyBP-C protein in a cat which was homozygous for the mutation. $\mathrm{Y} 2 \mathrm{H}$ analysis of the interaction between actin and the $\mathrm{COCI}$ domain in CMyBP-C revealed a 70\% reduction in B-galactosidase activity when the mutation was introduced, reflecting a decreased binding as a result of the amino acid substitution. Visualising of the CO domain showed that the substitution of the alanine for a proline in residue 31 was highly energetically unfavourable and resulted in only one hydrogen bond ( $\mathrm{H}$-bond) within the anti-parallel beta-strand instead of $2 \mathrm{H}$-bonds for alanine.

Conclusion: The P.3IP mutated CMyBP-C is expressed in cardiac tissue and the most likely pathogenic mechanism is interference with the assembly of the sarcomere due to reduced binding of the $\mathrm{COCl}$ domain of $\mathrm{CMyBP}-\mathrm{C}$ to actin.

\section{Two week proanthocyanidin treatment prevents heart failure in a rat model}

\author{
Maritza Kruger*, Sandrine Lecour" and Neil Davies* \\ "Hatter Institute for Cardiovascular Research, Department of Surgery, Groote Schuur Hospital and the University of Cape Town, \\ South Africa \\ \#Hatter Institute for Cardiovascular Research, Department of Medicine, Groote Schuur Hospital and the University of Cape Town, \\ South Africa
}

Heart failure is a multifactorial syndrome and a leading cause of mortality worldwide (Latronico et al., 2008; Mudd and Kass, 2008). To date, many pharmaceutical agents, such as angiotensin converting enzyme inhibitors and beta-blockers have been used to treat cardiac hypertrophy and early stages of heart failure. These therapies have proven reasonably effective; however, the incidence of mortality due to heart failure is still rising (De Luca et al., 2008; Travares et al., 2008; Tunuguntla 2007). Therefore, there is an important need for alternative therapeutic strategies, more specifically, natural therapies that is cost-effective and safe, to prevent or reverse hypertrophy before it develops into heart failure. Proanthocyanidolic oligomer supplementation (PCO), a grape seed extract, was shown to be effective in a skeletal muscle model of 
injury. In this model, PCO supplementation resulted in quicker muscle recovery following a contusion injury, favouring an anti-inflammatory state (Myburgh et al., 20 I I). Considering the positive effects of PCO on muscle recovery, as well as results suggesting that resveratrol, another member of the polyphenol family, could limit the occurrence of cardiac hypertrophy (Wojciechowski et al., 20 I0), the possibility exists that PCO might also be beneficial.

In the current study, an osmotic minipump containing isoproterenol $(2 \mathrm{mg} / \mathrm{kg} / \mathrm{day})$, a dual bl-b2-adrenergic receptor agonist, was used to induce heart failure. Male Wistar rats $(280$ - 320g) were orally gavaged with either PCO $(20 \mathrm{mg} / \mathrm{kg} /$ day $)$ or distilled water for 2 weeks prior to the subscapular implantation of the pump containing isoproterenol or ascorbate (vehicle). There were 8 animals in each of the 4 groups. After 7 days, the rats were killed and the heart isolated. Paraffin wax-embedded hearts were sectioned (2um) and stained with haematoxylin and eosin for the structural composition of the organs, as well as Massons' trichrome for fibrosis. An elastic neutrophil marker and a macrophage marker, EDI, were used to determine the infiltration of immune cells.

Results indicate that the isoproterenol groups lost significant amounts of weight I day $(p<0.05)$ after implantation compared to their controls. Histological analysis of hearts showed more damage, cardiomyocyte loss and fibrosis in the isoproterenol group receiving placebo treatment (I-PLA) compared to the group receiving PCO supplementation (I-PCO) group. Both groups displayed significantly more fibrosis than their respective controls. PCO treatment resulted in better recovery after isoproterenol infusion.

\section{Role of Toll-like receptor 4 in melatonin-induced cardioprotection}

\section{K.T. Lamont, L.H. Opie and S. Lecour}

Hatter Institute for Cardiovascular Research, Department of Medicine, Groote Schuur Hospital and the University of Cape Town, South Africa

Purpose: Melatonin, at concentration found in wine $(+/-75 \mathrm{ng} / \mathrm{L})$, confers cardioprotection against myocardial infarction via the activation of the SAFE (survivor activating factor enhancement) pathway that involves tumour necrosis factor (TNF) and signal transducer and activator of transcription 3 (STAT-3). However, the mechanism involved in melatonin-induced activation of TNF is unclear. As Toll-like receptors 4 (TLR4) can activate TNF in the cells, we propose that activation of the SAFE pathway with melatonin requires the activation of TLR4.

Methods: Isolated male Wistar rat hearts were perfused on a Langendorff system. The hearts were pre-treated with melatonin (75ng/L) with/ without TAK242 (500nM), an inhibitor of TLR4, and subjected to a 30 minute regional ischaemia followed by 2 hour of reperfusion. STAT-3 cardiomyocyte deficient (STAT3 -/-) mice, TNF deficient mice (TNF -/-) and their respective wildtype littermate controls were pre-treated with an activator of TLR4 or lipopolysaccharides (LPS, $0.2 \mathrm{mg} / \mathrm{kg}$, ip) $45 \mathrm{~min}$ prior to an ischaemia/reperfusion insult. Infarct size was determined as an endpoint.

Results: Control rat hearts subjected to ischaemia/reperfusion had an infarct size of $49.3 \pm 2.6 \%$. Melatonin reduced the infarct size to $14.9 \pm 1.5 \%$ ( $p<0.00$ I vs control). This protective effect was abolished in the presence of TAK inhibitor to $31.5 \pm 1.4 \%$ (ns. vs control). In wildtype mice, LPS improved infarct size from $40.6 \pm .3 .7 \%$ (controls) to $16.9 \pm 0.9 \%(\mathrm{p}<0.00 \mathrm{I})$ ). However, this protective effect was abolished in the STAT-3 -/- and TNF-/- mice.

Conclusion: Our data strongly suggest that TLR4 is required in melatonin-induced cardioprotection. Furthermore, TLR4 may act as an important component to activate the SAFE pathway with melatonin. 


\title{
Impact of inappropriate versus absolute or indexed left ventricular mass on on-treatment changes and community variations in ejection fraction
}

\author{
Carlos David Libhaber*, Angela J. Woodiwiss", Muzi J. Maseko*, Olebogeng H.I. Majane*, Aletta Esterhuyse*, \\ Richard Brooksbank*, Elena Libhaber", Pinhas Sareli* and Gavin R. Norton* \\ *Cardiovascular Pathophysiology and Genomics Research Unit, School of Physiology and School of Medicine Faculty of Health Sciences, \\ University of the Witwatersrand, Johannesburg, South Africa \\ \#Department of Medicine, Division of Cardiology, School of Medicine, Faculty of Health Sciences, Chris Hani Baragwanath Academic \\ Hospital, University of the Witwatersrand, Johannesburg, South Africa
}

Background: Whether the transition from left ventricular hypertrophy (LVH) to heart failure with a depressed ejection fraction (EF) represents a cause-effect relationship is unclear. LV mass (LVM) that exceeds that predicted from work load (inappropriate LVM [LVMinappr]), rather than LVH per se may cause a depressed EF.

Methods and results: In 168 mild-to-moderate hypertensives treated for 4 months with anti-hypertensive therapy and in 626 randomly selected participants over 16 years of age from a community sample using echocardiography, we assessed whether absolute LVM, LVM index (LVMI) or LVMinappr determine EF and the factors independently associated with LVMinappr. With adjustments for LV wall stress(s) and other confounders, whilst on-treatment decreases in LVM or LVMI were weakly related to an attenuated EF (partial $r=0.17, p<0.05)$ ontreatment decreases in LVMinappr were strongly related to increases in EF (partial $r=-0.38, p<0.000$ I) $(p<0.000$ I for comparison of partial $r$ values). Also with adjustments for $s$ and other confounders, in the community sample there was a strong inverse relationship between LVMinappr and EF (partial $r=-0.41, p<0.0001$ ), effects noted in both normotensives and hypertensives, whilst only modest inverse relations between LVM or LVMI and EF were noted (partial $r=-0.07$ to $-0.09, p<0.05-0.09)(p<0.000$ I for comparison of partial $r$ values). Although an increased LVMI was not associated with a reduced $E F(<55 \%)(p=0.50)$, a greater proportion of participants with an increased LVMinappr had a reduced EF than those with a normal LVMinappr (adjusted odds ratio $=6.85, \mathrm{p}<0.000 \mathrm{I}$ ). Obesity (mediated in-part through insulin resistance and inflammation [C-reactive protein]) was the principal factor that in-part accounted for variations in LVMinappr.

Conclusion: LVMinappr rather than absolute or indexed LVM is strongly related to changes in on-treatment EF, community variations in EF and the presence of a reduced EF. LVH can therefore be viewed as a compensatory change that preserves EF, but when in excess of that predicted by stroke work, a pathophysiological process causing a reduced EF.

\section{Potential mechanisms that account for obesity-related decreases in left ventricular diastolic function}

\author{
Carlos David Libhaber", Gavin R. Norton*, Olebogeng H.I. Majane*, Muzi J. Maseko*, Elena N. Libhaber", \\ Mohammed R. Essop", Pinhas Sareli* and Angela J. Woodiwiss* \\ "Cardiovascular Pathophysiology and Genomics Research Unit, School of Physiology and School of Medicine Faculty of Health Sciences, \\ University of the Witwatersrand, Johannesburg, South Africa \\ \#Department of Medicine, Division of Cardiology, School of Medicine, Faculty of Health Sciences, Chris Hani Baragwanath Academic \\ Hospital, University of the Witwatersrand, Johannesburg, South Africa
}

Introduction: Obesity predicts the development of heart failure independent of conventional risk factors. We have recently demonstrated in a community sample with a high prevalence of obesity that waist circumference has a strong and inverse relationship with early-to-atrial transmitral velocity (E/A), an index of diastolic function of the left ventricle (LV). The mechanisms that account for this relationship are unclear. Metabolic, neurohumoral, inflammatory (adipokines) or haemodynamic (central aortic blood pressures) factors may play a role.

Aims: To determine the relationship between transmitral E/A and indices of insulin resistance, measures of the circulating renin-angiotensinaldosterone system (RAAS), the inflammatory marker C-reactive protein (CRP), or central aortic BP in a community sample with a high prevalence of obesity. 
Methods: Transmitral E/A and left ventricular mass indexed for height ${ }^{7}$ were determined using echocardiography in 672 randomly selected participants of a community sample of African ancestry, $63 \%$ of whom were overweight or obese. Circulating RAAS and CRP concentrations were determined using immune radiometric techniques, a ${ }^{125}$ radio immune-assay, and a solid phase sandwich ELISA. Central aortic BP was assessed using radial tonometry and SphygmoCor software. Insulin resistance was evaluated from the homeostasis model (HOMA-IR) as determined from fasting insulin and glucose concentrations.

Results: Independent of a number of confounders including age, gender, pulse rate, conventional diastolic (or systolic) BP, anti-hypertensive treatment, left ventricular mass index (LVMI) and the presence of diabetes mellitus or an $\mathrm{HbAI}>$ 6. I\%; waist circumference was independently and inversely associated with E/A $(P<0.0005)$. Similarly, independent of confounders HOMA-IR was independently associated with E/A $(p<0.01)$, However, despite CRP being strongly associated with indices of excess adiposity, neither measures of the RAAS, nor CRP were independently associated with E/A ( $p=0.376-0.73)$ and central aortic BP was not associated with E/A independent of conventional BP.

Conclusion: Insulin resistance may be an indicator, but neither RAAS obesity-associated inflammatory changes nor central aortic BP can explain the relationship between obesity and a reduced LV diastolic function beyond conventional cardiovascular risk factors or LVMI.

\title{
Improved survival and quality of life after TAVI
}

\author{
Clinton Lloyd, Ian Cox, Rashmi Birla, Sam Melhuish, Tania Riches and Malcolm Dalrymple-Hay \\ Derriford Hospital, Plymouth, Devon, United Kingdom
}

Introduction: Transcatheter aortic valve implantation (TAVI) is a new modality of treatment for severe symptomatic aortic stenosis patients who are considered to be at high risk for conventional open-heart surgery. The aim of this study was to compare survival with medical therapy and open-heart surgery and to assess quality of life $(\mathrm{QOL})$ in patients who underwent TAVI.

Method: I 50 patients reviewed for possible TAVI were assigned to TAVI (8I), open surgery ( I3) or medical therapy (56). Additionally, 30 of the patients receiving TAVI were interviewed pre-operatively using a questionnaire of 15 questions (designed locally) to compare pre- and post- operative quality of life, covering aspects of general health, routine physical functioning and essential daily activities (scoring 0-5).

Results: Overall survival (Log-rank Test $\mathrm{p}<0.05$ ) for TAVI patients (4 deaths) was better than open surgery (2 deaths) or medical therapy (7 deaths). 24 patients completed the QOL survey ( 12 Male). Mean age 80.29 years (range 56 - 89 years) 2 failed to respond but are known to be alive, 2 patients died post-operatively and 2 declined follow-up. Mean scores for General Health (GH), Physical Functioning (PF) and Essential Daily Activities (EDA) showed significant improvement post-operatively (39.58 \pm 9.23 vs $49.50 \pm 7.21$ ) $p=<0.00$ I in TAVI survivors. Conclusion: In this cohort of the population that tend to have multiple co-morbidities, the overall quality of life improves significantly after TAVI. The cost benefit and long term outcome have not been fully assessed in this study, however, TAVI should be considered a viable option for patients with severe symptomatic aortic stenosis who are high risk for conventional surgery.

\section{Isolated left ventricular non-compaction (ILVNC): the Chris Hani Baragwanath experience}

\author{
Nirvarthi Maharaj, Ferande Peters, Farouk Mamdoo, Claudia dos Santos, Hiral Matioda, Elena Libhaber and \\ Mohammed R. Essop \\ Department of Medicine, Division of Cardiology, Chris Hani Baragwanath Academic Hospital, University of the Witwatersrand, \\ Johannesburg, South Africa
}

Introduction: Isolated left ventricular non-compaction (ILVNC) is thought to occur as a result of failure of myocardial compaction during embryogenesis. Heart failure, arrhythmias and thrombo-embolism are the cardinal clinical manifestations of this disorder. Published data on the clinical and echocardiographic features of ILVNC in Africans is limited. We sought to evaluate the clinical and echocardiographic features of African patients with ILVNC.

Methods: This ongoing prospective single centre study enrolled patients attending Chris Hani Baragwanath Cardiac Clinic who satisfied the Jenni echocardiographic criteria for the diagnosis of ILVNC. Exclusion criteria were hypertension, coronary artery disease, organic valvular 
disease and any systemic illness that could contribute to left ventricular dysfunction. The control population comprised $4 \mathrm{I}$ age and sex matched normal individuals. Transthoracic echocardiography was performed according to the American Society of Echocardiography (ASE) guidelines 2006 and ASE 2010 guidelines on right heart assessment to determine ventricular structure and function. Clinical and echocardiographic data were compared between patients with ILVNC and the control population.

Results: The mean age was $44.25 \pm 13.47$ years with $56 \%$ female in the ILVNC group $(n=72)$. Heart failure was the most frequent clinical manifestation of patients with ILVNC and occurred in 70 (97.2\%) of subjects. On standard medical therapy which included beta blockers and ACE inhibitors most subjects were NYHA 2 (57.1\%). The mean left ventricular end diastolic diameter was $59.5 \pm 8.33 \mathrm{~mm}$ with ejection fraction $28.16 \pm 11.85 \%$. The right ventricle was found to be dysfunctional in 44 (61.1\%) of patients with ILVNC with the mean RV S' (9.32 $\pm 2.79 \mathrm{~cm} / \mathrm{s}$ ) significantly lower than that of the control group. Moderate or severe tricuspid regurgitation was documented in 48 (66.6\%) of subjects. The systolic pulmonary artery pressure was measured in 62 (86.1\%) of subjects with a mean $39.44 \pm 9.72 \mathrm{mmHg}$. No echocardiographic features suggestive of ILVNC were noted in a healthy control group of African descent.

Conclusion: ILVNC is not infrequent in the South African Black population. ILVNC in patients of African descent can be characterised by biventricular dysfunction and/or isolated left-sided dysfunction and heart failure as a result of systolic dysfunction is the most common clinical presentation.

\section{Left ventricular twist abnormalities in isolated left ventricular non-compaction (ILVNC)}

\section{Nirvarthi Maharaj, Ferande Peters, Elena Libhaber, Claudia dos Santos, Hiral Matioda and Mohammed R. Essop}

Department of Medicine, Division of Cardiology, Chris Hani Baragwanath Academic Hospital, University of the Witwatersrand, Johannesburg, South Africa

Introduction: Left ventricular twist is defined as the wringing motion of the heart during systole where the LV apex moves in a counterclockwise direction while the base moves in a clockwise direction. LV twist is an important factor contributing to the efficiency of the ejection of blood during systole. Isolated left ventricular non-compaction (ILVNC) is thought to occur as a result of failure of myocardial compaction during embryogenesis. There is a paucity of data assessing myocardial mechanics in African patients with ILVNC. We sought to evaluate left ventricular twist mechanics in subjects with ILVNC in whom heart failure as a result of left ventricular systolic dysfunction is the major clinical manifestation.

Methods: This prospective single centre study enrolled 72 patients attending Chris Hani Baragwanath Cardiac Clinic between July 2009 and December 201 I who satisfied the Jenni echocardiographic criteria for the diagnosis of ILVNC. The control population comprised 39 age and sex matched unrelated normal individuals. Parasternal short-axis images at LV basal and apical level were obtained with 3 consecutive endexpiratory cardiac cycles. Data was transferred to a QLAB workstation (QLAB Advanced Quantification Software version 8.0, Philips) for off-line analysis. Clockwise rotation was presented as a negative value and counter clockwise rotation as a positive value when viewed from the apex. Peak apical (AR), basal rotation at a time isochronous with peak apical rotation (BR) and Net Twist (defined as the maximal value of instantaneous $A R-B R$ ) were measured. Rigid body rotation (RBR) was defined as AR and BR occurring in the same direction.

Results: Of 72 ILVNC patients, 3 I (43.1\%) had a normal twist pattern, while 4 I (56.9\%) exhibited RBR. In the group with RBR, 27 (65.9\%) patients had clockwise rotation and 14 (34.1\%) patients had counter-clockwise rotation. In the 31 patients with ILVNC and a normal twist pattern, $\operatorname{AR}(1.75+1.45$ vs. $4.96+2.99)$ and $B R(-1.17+1.15$ vs. $-2.98+1.63)$ as well as net twist $(3.1+1.71$ vs. $7.94+3.35)$ were all diminished compared to the control group $(p<0.000 \mathrm{I})$.

Conclusion: LV twist mechanics is impaired in African patients with ILVNC. RBR occurs in $56.9 \%$ of patients and can be either in a clockwise or counter-clockwise direction. 
Twist mechanics in African hypertension patients with systolic dysfunction: an analysis using speckle tracking echocardiography

\section{Nirvarthi Maharaj, Ferande Peters, Elena Libhaber and Mohammed R. Essop}

Department of Medicine, Division of Cardiology, Chris Hani Baragwanath Academic Hospital, University of the Witwatersrand, Johannesburg, South Africa

Introduction: Speckle tracking evaluating twist mechanics has emerged as a sensitive quantitative technique in assessing left ventricular (LV) function. LV twist describes the net rotational difference of the apex with respect to the base of the heart and has an important role in LV function. Hypertension associated changes in LV twist have not been previously evaluated in African patients with LV systolic dysfunction in the absence of coronary artery disease. This study evaluates LV twist dynamics in African hypertensive patients with systolic dysfunction.

Methods: 4 I consecutive hypertensive patients (HTLEF) from the Chris Hani Baragwanath Cardiac clinic between January $201 \mathrm{I}$ to January 2012 with ejection fraction less than $45 \%$ on echocardiography and normal coronary angiography were recruited. The inclusion criteria were: sinus rhythm, age $>30$, no diabetes and normal renal function. The control population comprised 4 I normal individuals. To optimise speckle tracking, two-dimensional gray-scale harmonic images were obtained at a frame rate of 60 to 80 frames/s. Parasternal short-axis images at LV basal and apical level were obtained with 3 consecutive end-expiratory cardiac cycles. Data was transferred to a QLAB workstation (QLAB Advanced Quantification Software version 8.0, Philips) for off-line analysis. Clockwise rotation was presented as a negative value and counter clockwise rotation as a positive value when viewed from the apex. Peak apical (AR), basal rotation at a time isochronous with peak apical rotation (BR) and Net Twist (defined as the maximal value of instantaneous AR-BR) were measured.

Results: In the HTLEF group, the mean age was 55.0 +/- 9.1 years with 22 (54\%) females. The HTLEF patients had a mean duration of hypertension of $12.2+/-6.5$ years with $3.2+/-1.6$ years of heart failure. Ejection fraction in the HTLEF group was $33.0+/-7.6 \%$. LV twist and rotation at apical and basal planes were lower in patients with (HTLEF) $(1.89+/-0.99$ vs. 7.15 +/- 2.26; $p<0.000$ I for AR; - I.44 +/- 0.78 vs. -3.75 +/- I.6I; $p<0.000$ I for BR; 3.3 +/- I. 17 vs. 10.9 +/- 2.7; $p<0.000$ I for NET TWIST) compared to the control group.

Conclusion: Left ventricular twist is diminished in African hypertensive patients with systolic dysfunction. The twist is reduced as a result of both diminished apical and basal rotation.

\section{Defensive active coping (AC) facilitates time-domain HRV and sub-clinical vascular disease: the sympathetic activity and ambulatory blood pressure in Africans (SABPA) study}

\section{Leoné Malan", Mark Hamer", Markus Schlaich ${ }^{\dagger}$, Gavin Lambert ${ }^{\dagger}$, Manja Reimann ${ }^{\ddagger}$, Nancy Frasure-Smith ${ }^{\dagger}$, Lisa Uys*,} Andrea de Kock* and Nico Malan*

"Hypertension in Africa Research Team (HART), North West University, Potchefstroom Campus, South Africa

\#Department of Epidemiology and Public Health, University College of London, UK

tDepartment of Biological Psychology, Neurovascular Hypertension \& Kidney Disease and Human Neurotransmitters Laboratories, Baker IDI Heart and Diabetes Institute, Melbourne, Australia

*Department of Neurology, Medical Faculty Carl Gustav Carus, Dresden University of Technology, Germany

•Department of Psychiatry and School of Nursing, McGill University, Centre Hospitalier Université de Montréal and Research Centre, Montreal Heart Institute, Canada

Objectives: Defensive active coping (AC) responses in urban African males have been associated with vascular responsiveness partly explaining autonomic nervous system dysfunction. We therefore aimed to assess if AC responses facilitate early sub-clinical vascular disease via alterations in time-domain heart rate variability (HRV).

Methods: We included 363 African and Caucasian men and women without pre-existing atrial fibrillation, aged $45 \pm 9$ years. The Coping Strategy Indicator stratified ethnic gender groups. We collected B-mode ultrasound carotid intima media thickness far wall (CIMTf), 24h BP and time-domain HRV or standard deviation of all NN intervals (SDNN). Overnight fasting blood was obtained. 
Results: Overall Africans and AC Africans, mostly men, revealed $(p=0.05)$ a poorer lifestyle profile and higher prevalence of hypertensive status compared to their Caucasian counterparts. Moderately depressed HRV (SDNN <100ms) was prevalent in 32\% of Africans compared to I5\% of Caucasians. A similar trend was shown for the AC African participants (32\%) in comparison to Caucasians (I6\%). Depressed HRV was associated with 24h SBP and DBP in AC African men only. Only their SBP values predicted structural vascular changes.

Conclusion: Depressed HRV was associated with increased 24h BP in AC African men and women. Defensive AC facilitated autonomic nervous system dysfunction, acting as a hyperkinetic driving force for increased 24h SBP and early sub-clinical vascular changes in the Africans.

\section{The utility of transoesophageal echocardiography in young stroke patients: insights from TORCH}

\section{Farouk Mamdoo, Ferande Peters, Anupa Patel, Hiral Matioda, Samantha Govender and Mohammed R. Essop}

Department of Medicine, Division of Cardiology, Chris Hani Baragwanath Academic Hospital, University of the Witwatersrand, Johannesburg, South Africa

Background: The prevalence of a cardiac source for cerebral embolism in young stroke patients in South Africa is unknown. Prior publications have documented greater frequency of stroke in young patients with HIV and the frequency of cardiac abnormalities in these patients is unclear. Transoesophageal echocardiography (TEE) with contrast enhancement has a high sensitivity for detecting potential cardiac sources of cerebral embolism. The Transoesophageal Registry of Chris Hani Baragwanath Academic Hospital (TORCH) was established in 2010 and entails prospective data collection on all TEE examinations performed at this hospital.

Aim: To examine the prevalence of cardio-embolism with TEE in young patients with acute ischaemic stroke.

Methods: Retrospective analysis of the TORCH data on 64 patients with young stroke (age <40 years) who presented from June 2010 to January 2012. Patients were referred for evaluation for possible cardiac source of embolism from within $\mathrm{CH}$ Baragwanath Academic Hospital and referring hospitals. Inclusion criteria were (I) Confirmed ischaemic stroke on brain imaging (2) No obvious cause for stroke on ECG, transthoracic echocardiography, carotid Duplex studies and haematologic evaluation (3) No evidence of meningitis/encephalitis on lumbar puncture. HIV status was documented in each patient after obtaining informed consent. All patients underwent transthoracic echocardiographic imaging and where no obvious cardiac source was identified they were referred for TEE. TEEs were performed under conscious sedation using the X7-2T TEE transducer coupled to a Philips iE-33 machine and all data were analysed on QLAB software linked to an Excelera system. A bubble test was performed in all subjects using agitated saline contrast (ASC) via a peripheral vein and recordings taken within an 8 beat cycle with either a Valsalva manoeuvre or firm pressure applied over the patient's abdomen. A positive bubble study was considered positive with the appearance of ASC in the left-sided chambers within 4 beats of the infusion in appropriately selected views. 3DTEE was utilised when deemed necessary and in particular for viewing the atrial septum.

Results: Of the 64 patients, 53 (83\%) tested positive for HIV infection. Overall 5 (8\%) patients had a potential source for cardio-cerebral embolism: I patient had an isolated patent foramen ovale, 2 had small secundum atrial septal defects and 2 had atrial septal aneurysms ( 1 of which had an associated patent foramen ovale). It was unclear whether these defects could clearly be linked to the presenting stroke.

Conclusion: The diagnostic yield of TEE in young stroke patients in a high HIV prevalence area is low. Even in the small number of patients in whom structural abnormalities are identified, it remains unclear whether these defects clearly account for the stroke. Based on these findings, we cannot recommend routine TEE in young stroke patients in areas of high HIV prevalence. 


\title{
Coronary artery disease prevalence amongst patients undergoing valve replacement surgery
}

\section{Ruchika Meel, Ahmed R. Essop, Anthony Becker, Chris Zambakides, Anthony Yip, Richard Nethononda and Mohammed R. Essop}

Department of Medicine, Division of Cardiology, Chris Hani Baragwanath Academic Hospital, University of the Witwatersrand, Johannesburg, South Africa

Introduction: The prevalence of coronary artery disease (CAD) amongst patients presented for valve surgery has important implications for routine angiography. According to the 2006 ACC/AHA guidelines on valve disease, coronary angiography is recommended in symptomatic men >35 prior to aortic valve replacement; any patient >35 with 2 or more coronary risk factors; asymptomatic men >45; and women $>55$. However, in a large Brazilian study no asymptomatic patient, <60 years, without risk factors was found to have CAD. The prevalence of CAD, in predominantly black patients, presented for valve surgery in South Africa has not been published.

Methods: A prospective, descriptive study of patients presented for valve surgery between 2010 and $201 \mathrm{I}$ was performed. CAD was defined as stenosis of $70 \%$ or greater, in one or more epicardial vessels as defined by quantitative coronary angiography.

Results: A total of 116 patients were included. Of these $56.9 \%$ were female and the mean age was 57.4 years. Black patients represented 66.4\%; Caucasians 19.8\%; and, coloured and Indian patients 13.8\%. Hypertension and smoking were the most common risk factors (26.7\% and 16.4\%, respectively). Diabetes mellitus, dyslipidaemia, chronic kidney disease and prior CAD occurred in 15.5\%. The remainder had no known risk factors. Angina was present in 18\% of patients. New York Heart Association functional Class 2, 3 and 4 patients were distributed as follows: 54\%, 38.8\% and $0.7 \%$, respectively. HIV prevalence was 12\% - half of them were on anti-retroviral therapy. An isolated valve lesion occurred in $69 \%$ of patients with the remainder having 2 or more lesions. The most common valve lesion was aortic stenosis (43. $1 \%$ ), followed by mitral stenosis (36.2\%), aortic regurgitation (29.3\%), mitral regurgitation (25.9\%) and tricuspid regurgitation (19\%). The most common aetiology was rheumatic heart disease (58.6\%), followed by degenerative valve disease (24.1\%). Congenital valve disease, infective endocarditis and ascending aorta aneurysms accounted for the remainder (17.2\%). CAD was documented in 10 patients (8.6\%), of whom 8 had single vessel disease and 2 had double vessel disease. The age range was 55 to 87 . Only one black patient, aged 67, had CAD. None were HIV positive, 4 had preceding angina and 4 patients had no risk factors for CAD.

Conclusion: The prevalence of CAD in patients presented for valve surgery in this study was $8.6 \%$. For asymptomatic, black patients without risk factors, the need for routine angiography prior to valve replacement is questionable.

\section{Cardiological family screening in isolated left ventricular non-compaction (ILVNC)}

\author{
Michael Thamaga Mogogane, Ferande Peters, Claudia dos Santos, Hiral Matioda, Richard Nethononda and Rafique Essop \\ Department of Medicine, Division of Cardiology, Chris Hani Baragwanath Academic Hospital, University of the Witwatersrand, \\ Johannesburg, South Africa
}

Introduction: Isolated Left ventricular non-compaction (ILVNC) is presumed to be a genetic cardiomyopathy with familial and sporadic forms. Familial disease is thought to occur in 15 to $50 \%$ of adults. Familial cases of ILVNC display considerable genetic heterogeneity. In Africa there is a paucity of data on cardiological family screening among families of patients diagnosed with ILVNC.

Methods: Systematic cardiological screening of first degree relatives of patients diagnosed with ILVNC commenced in December 201 I at the Chris Hani Baragwanath cardiomyopathy clinic. First degree relatives were evaluated by 2 clinicians (M.M. and F.P.). Evaluation included a medical history, physical examination, electrocardiography and echocardiography. ILVNC was diagnosed based on the Jenni echocardiography criteria. Cases with poor echo windows or equivocal echocardiographic features were referred for cardiac MRI.

Results: 35 relatives from I I families were screened. The mean age of relatives was 26.8 years (range 2 to 73 years), 26 (74\%) were female. ILVNC was diagnosed in 8 (22.8\%) participants from 5 unrelated families. All 8 (22.8\%) of the relatives with ILVNC were NYHA I and all had a normal left ventricular ejection fraction. In all 8 affected individuals the apex and mid lateral walls displayed features of non-compaction. No relatives screened displayed echocardiographic features of dilated or hypertrophic cardiomyopathy. Two relatives with equivocal echo- 
cardiographic features for ILVNC were identified and are awaiting elective cardiac MRI. Four (50\%) of the 8 affected family members had normal electrocardiograms, while the other 4 had nonspecific ST- and T-wave abnormality.

Conclusion: These preliminary results reveal that cardiological family screening identified ILVNC in $22.8 \%$ of relatives, the majority of whom were asymptomatic with preserved ejection fraction. More extensive cardiological screening of a larger cohort of ILVNC relatives is required to determine more accurately the prevalence of familial involvement. Cases identified as ILVNC during screening require long term follow-up to determine the clinical outcome and prognosis of these individuals.

\title{
Mortality associated with infective endocarditis (IE)
}

\section{Jameel Moosa, Ngoba Tsabedze, Ahmed Vachiat, Rohan Ramjee, Thomas Kalk, Keir McCutcheon, Lance Mkhwanazi, Riaz Garda and Pravin Manga}

Division of Cardiology, Charlotte Maxeke Johannesburg Academic Hospital, University of the Witwatersrand, Johannesburg, South Africa

Introduction: Infective endocarditis (IE) in the post-antibiotic era is associated with an in-hospital mortality rate of 18 - $23 \%$ in well developed countries. The mean age of these patients is 60 years mainly due to a higher prevalence of degenerative cardiac disease and a lower incidence of rheumatic heart disease (RHD). The aim of our study was to assess the in-hospital mortality rate of infective endocarditis in a busy public academic hospital in South Africa.

Methods: A retrospective analysis of all patients with probable or definite IE presenting to CMJAH from 2006 to 201 I was performed.

Results: A total of 101 patients with IE were reviewed. The mean age of this study population was 38 years with 62 males (6I\%) and 39 females (39\%). The mortality rate in the overall cohort was 28\% (28 patients). The mean age of this group was 39.8 years. Blood cultures were positive in 14 patients (48.3\%), with majority being Staphylococcus aureus (35.7\%), Streptococcus viridans (2I.4\%) and Coagulase negative Staphylococcus (CNS) (14.3\%). The mitral valve alone was involved in 13 patients (47\%), mixed mitral/aortic valve in 8 patients (28\%), isolated aortic valve in 5 patients ( $18 \%$ ) and 2 patients (7\%) had isolated tricuspid valve (TV) endocarditis. Fifteen (54\%) of these patients had RHD, 4 (13.8\%) had degenerative valves, I (3.4\%) had MV prolapse, 2 (7\%) had prosthetic valves and 6 (21\%) had no predisposing valve pathology. Of the 73 surviving patients, the mean age was similar at 36.9 years. Blood cultures were positive in 28 patients (38\%), with the majority being Streptococcus viridans (35.7\%), Staphylococcus aureus (14.3\%) and CNS (10.7\%). The mitral valve alone was involved in 34 patients (46\%), isolated aortic valve in 18 (25\%), mixed mitral/aortic valve in 16 patients (22\%) and 5 patients (7\%) had isolated TV endocarditis. The majority 45 (61\%) of these patients had RHD.

Conclusion: The mortality rate of IE in our unit over the last 6 years is higher than that found in developed countries. The mean age of our patients is significantly younger as RHD is still the most prevalent predisposing factor. Staphylococcus aureus endocarditis was significantly more prevalent among non-survivors.

\section{Invasive haemodynamic evaluation of category I pulmonary arterial hypertension (PAH)}

\author{
Muhammad Zaid Moosa", Paul Williams", Ahmed R. Essop* and Mohammed R. Essop* \\ *Department of Medicine, Division of Cardiology, Chris Hani Baragwanath Academic Hospital, University of the Witwatersrand, \\ Johannesburg, South Africa \\ \#Pulmonary Hypertension Research Group, Division of Cardiology, University of Witwatersrand, Milpark Hospital, Johannesburg, South Africa
}

Introduction: Pulmonary arterial hypertension $(\mathrm{PAH})$ is a rare disorder which may be fatal if not diagnosed early. The haemodynamics of PAH have not been well documented in South Africa and no data on vasoreactivity testing exists.

Aim: To document in detail the invasive haemodynamic indices in 14 patients referred for investigation of category I PAH.

Methods: Patients with confirmed category I PAH were included in the study. A right and left heart catheterisation was performed together with measurement of cardiac output using the Fick method. Vascular resistances were calculated and response to Nitric Oxide evaluated. A positive vasodilator response was defined as a $20 \%$ reduction in mean Pa pressure to an absolute value of $<40 \mathrm{mmH}$. 
Results: The mean age of the cohort was $37.6 \pm 12.6$ years. The etiology of PAH in the group was found to be idiopathic in II patients (78.6\%), HIV associated in 2 patients (14.3\%) and secondary to a congenital shunt in I patient (7.1\%). The mean PA pressure for the group was markedly elevated $(103 / 46 / 68 \mathrm{mmHg})$ with significant reduction in cardiac index $\left(2.1 \pm 1.1 \mathrm{~L} / \mathrm{min}^{2} \mathrm{~cm}^{2}\right)$. Pulmonary vascular resistance was high ( $8 \pm 10$ Wood units) and although it decreased following NO ( $4 \pm 12$ Wood units), there was no significant change in the ratio of PVR:SVR ( $0.72 \pm 0.28$ pre versus $0.66 \pm 0.3$ post). Only I patient was classified as a responder to NO.

Conclusion: Patients in this study presented with advanced pulmonary arterial hypertension and the majority showed no reversibility to Nitric Oxide. These data would indicate a need for greater awareness and earlier referral to specialised centres in order to improve the outcome in these patients.

\title{
The distribution of conventional pulsed wave-tissue Doppler imaging measurements of the right ventricle by age and sex in a healthy Black South African population
}

\author{
Muhammad Zaid Moosa, Ferande Peters, Nirthi Maharaj and Mohammed R. Essop \\ Department of Medicine, Division of Cardiology, Chris Hani Baragwanath Academic Hospital, University of the Witwatersrand, \\ Johannesburg, South Africa
}

Introduction: Because of its complex shape and marked load dependence, the study of the RV remains challenging. Pulse wave tissue Doppler imaging (PW-TDI) of the lateral tricuspid annulus allows non-invasive quantitative assessment of RV systolic and diastolic function in several cardiac disorders and has a good correlation with invasive haemodynamic indices and RV ejection fraction by CMRI. Small population-based studies in the Western world have been undertaken to determine reference values of RV myocardial function using tissue Doppler imaging in healthy individuals. Currently there are no normative reference values for these clinically important parameters in patients of African ethnicity. Aim: To determine PW -TDI measurements of the RV in a healthy black South African cohort.

Methods: A total of 100 healthy subjects aged 18 to 64 years with normal left and right sided chamber dimensions and function assessed by echocardiography were evaluated. The subjects were subsequently divided into 3 age groups: 18 to 29, 30 to 44 and >45 years. PW-TDI was measured from standard apical 4-chamber views by sampling at the junction of the RV free wall and insertion point of the tricuspid leaflets to the lateral RV annulus according to ASE/EAE recommendations. All studies were performed by 2 experienced sonographers and measurements of myocardial Systolic velocity ( $\left.S^{\prime}\right)$, Early diastolic velocity ( $\left.E^{\prime}\right)$ and Atrial diastolic velocity ( $A^{\prime}$ ) were interpreted offline by a single cardiologist. Results: The mean age of the cohort was $37.45 \pm 12.32$ years of whom 68\% were female. Averaged PW-TDI values for the entire population were $S^{\prime}-12.84 \pm 2.3 ; E^{\prime}-11.61 \pm 3.4$ and $A^{\prime}-10.74 \pm 3.3 \mathrm{~cm} / \mathrm{s}$. Analysis of PW-TDI for the 3 age groups revealed the following: With an increase in age the $S^{\prime}$ decreased ( $R$ value: $\left.0.41, p=0.69\right)$, the $A^{\prime}$ increased $(R$ value: $0.41, p=0.00 I)$, and the $E^{\prime}$ decreased $(R$ value: -0.35 , $\mathrm{p}=0.00 \mathrm{I}$ ) - the latter two showing moderate correlation and statistical significance.

Conclusion: The reference ranges presented for TDI parameters S', E` and A' velocities in Black South African individuals will help standardise the assessment of RV function by tissue Doppler echocardiography and have implications in diagnosing RV dysfunction in these patients. A correlation exists between age and diastolic TDI velocities.

\section{Non-ST elevation myocardial infarction (NSTEMI) in 3 hospital settings in South Africa. Does level of care influence management and outcome? A retrospective cohort study}

\author{
J. Moses*, A.F. Doubell, P.G. Herbst*, K.J.C. Klusmann" and H.S.V.H. Weich* \\ "Division of Cardiology, Department of Medicine, University of Stellenbosch and Tygerberg Hospital, South Africa \\ \#Department of Medicine, Worcester Hospital, Worcester, South Africa
}

Introduction: International guidelines state that patients presenting with an acute coronary syndrome (ACS) without ST-elevation but with raised markers of myocardial damage (NSTEMI) should receive early angiography and revascularisation if possible. Current guidelines for the secondary hospitals in the Western Cape dictate that patients with NSTEMls be admitted for conservative medical management and 
only referred for early angiography if haemodynamically unstable or experiencing ongoing ischaemia (chest pain or dynamic ECG changes). Troponins are taken for prognostication 6 - 12 hours after the index event. The aim is to identify a group of very high risk patients who are referred for early angiography. The remaining patients receive standard medical treatment and if they remain stable and do not reveal increased risk during pre-discharge risk-stratification, are referred to the tertiary centre as outpatients for possible angiography. We aimed to record if patients presenting to secondary hospitals are less likely to receive early invasive management and to determine if this exposed some patients to increased risk.

Methods: A retrospective cohort study was conducted assessing adult patients presenting with NSTEMls to Tygerberg Hospital (TBH), Karl Bremer Hospital $(\mathrm{KBH})$ and Worcester Hospital $(\mathrm{WH})$ over a one-year period from September 2010 to August 20I I. The data was analysed as 2 cohorts, a secondary hospital cohort $(\mathrm{KBH}$ and $\mathrm{WH})$ and a tertiary hospital cohort $(\mathrm{TBH})$. Where differences were found between the 2 groups, a sub-analysis was done comparing the $\mathrm{WH}$ group and the $\mathrm{KBH}$ group.

Results: The $\mathrm{TBH}$ and secondary hospital groups were similar in terms of baseline characteristics and clinical presentation. The management of the 2 groups differed in that those cases presenting to TBH were more likely to receive early ß-blockade $(67 \%$ vs $35 \%, p=0.0055)$ and in-patient coronary angiography ( $94 \%$ vs $51 \%, p=<0.000 \mathrm{I}$ ). There was no difference in management between the $\mathrm{KBH}$ and $\mathrm{WH}$ groups in sub-analysis. The TBH patients had a better in-patient mortality ( $6 \%$ vs $23 \%, p=0.0326$ ) than the secondary hospital group.

Conclusion: This study confirmed our hypothesis that management of a NSTEMI differs between hospitals in the public health sector depending on the level of service to which the patient first presents.

\section{The role of novel protein-protein interactions in the function and mechanism of the sarcomeric protein, myosin binding protein H (MyB PH)}

\section{Jacoba Martina Mouton*, Johanna C. Moolman-Smook" and Craig J. Kinnear"}

"Department of Biomedical Sciences, Faculty of Health Sciences, University of Stellenbosch, South Africa

\#University of Stellenbosch Medical Research Centre (US/MRC), Centre for Molecular and Cellular Biology,

Department of Biomedical Sciences, University of Stellenbosch, South Africa

Introduction: Left ventricular hypertrophy $(\mathrm{LVH})$ is acknowledged as a major risk factor for cardiovascular morbidity and mortality and is a feature of many common diseases, such as hypertension and diabetes. It is therefore, important to understand the underlying mechanisms influencing the development of hypertrophy. Hypertrophic cardiomyopathy (HCM) has been viewed as a model disease in which to study the causal molecular factors underlying isolated cardiac hypertrophy. HCM is a heterogeneous disease that manifests with various phenotypes and clinical symptoms, even in families with the same genetic defects. HCM is classically described as a disease of the sarcomere, which, as part of myofibrils, constitutes the contractile elements in cardiac muscle. One of the regions of the sarcomere that has been identified as playing a key role in the regulation of contractility is the C-zone. Identifying binding partners of one of the C-zone proteins, myosin binding protein $\mathrm{C}$ (MyBPC), has led to insights into the function of this protein. However, myosin binding protein $\mathrm{H}(\mathrm{MyBPH})$ is another member of the myosin binding protein (MyBP) family, located within this region, of which very little is known.

Given the sequence homology and similarity in structure between MyBPC and MyBPH, and since both proteins are known to bind myosin, we propose that $\mathrm{MyBPH}$ may play critical roles in the structure and functionality of the cardiac sarcomere and possibly in HCM pathogenesis.

Methods: The present study reports the identification of interacting partners of $\mathrm{MyBPH}$ with the aim to identify the role of this protein in the sarcomere, using yeast two-hybrid $(\mathrm{Y} 2 \mathrm{H})$ analysis.

Results: Twelve interacting partners were identified of which 3 (SUMO-conjugating enzyme UBC9, alpha cardiac actin (ACTC), myosin 7 (Myh7)) were considered putative physiological interactors based on the plausibility of the interactions as assessed in silico. These interactions will be verified using a number of independent molecular techniques that co-immunoprecipitation and three-dimensional, live cell fluorescencebased co-localisation.

Conclusion: The results of this study provide important clues to the function of $\mathrm{MyBPH}$ and, in so doing, improve our knowledge and understanding of this protein's role in the cardiac sarcomere. 


\title{
Assessing the effect of a hypertrophic cardiomyopathy causing mutation in cardiac Troponin I on the binding affinity of two known interactors of cardiac Troponin I
}

\author{
Annika Neethling*, Jacoba Martina Mouton* and Craig Kinnear" \\ "Department of Biomedical Sciences, Faculty of Health Sciences, University of Stellenbosch, South Africa \\ \#University of Stellenbosch Medical Research Centre (US/MRC), Centre for Molecular and Cellular Biology, \\ Department of Biomedical Sciences, University of Stellenbosch, South Africa
}

Introduction: Cardiac troponin I (cTnl), encoded by the sarcomeric gene TNNI3, is known to be the inhibitory component in the troponin complex and bind to cardiac actin (ACTC) and cardiac troponin C (CTNC) in the sarcomere. This subunit (cTNI) is essential in controlling heart muscle contraction in association with intracellular calcium ion levels. Previous studies have provided evidence that mutations in TNNI3 lead to the development of Hypertrophic cardiomyopathy (HCM), an autosomal dominantly inherited cardiac muscle disease that is becoming more common among the general population, affecting 1:500 individuals. In a recent investigation, we identified a novel HCM- causing mutation in the cardiac troponin I (CTNI) encoding gene (TNNI3). This mutation resulted in the substitution of a Leucine for a Histadine at amino acid I $44(\mathrm{LI} 44 \mathrm{H})$ which is located in the inhibitory region of CTNI. This region contains binding sites for both ACTC and cTNC. The aim of the present study is to investigate whether the $\mathrm{LI} 44 \mathrm{H}$ mutation has any effect on the ability of CTNI to bind ACTC and CTNC.

Methods: Yeast-two-hybrid (Y2H) based ONPG Quantitative B-Galactosidase assays were used to assess the binding affinity of both wildtype and mutant CTNI (LI44H) constructs for its known protein interactors, ACTC and cTNC. Statistical analysis was performed using the Graphpad Prism ${ }^{\mathrm{TM}}$ statistical software package.

Results: The results indicated that the $\mathrm{L} \mid 44 \mathrm{H}$ mutation significantly strengthened the interaction between $c T N I$ and $A C T C$ ( $P=0.0002$ ), suggesting that the mutation may result in a more tightly assembled sarcomere, which could lead to significant impairment of function. No significant difference was observed for the binding affinity of wildtype and mutant constructs to cTNC $(p=0.6077)$.

Conclusion: In the present study in vitro experiments demonstrated that the binding of CTNI to ACTC is significantly increased, which may lead to a decrease in the inhibition of cardiac contraction. We conclude that the LI44H mutation may cause HCM through impaired relaxation rather than impaired contraction.

\section{The role of the novel biomarker ST2 as a marker of left ventricular remodelling in a native African Hypertensive Cohort}

\author{
Dike Ojji", L. Lacerda\#, S. Lecour", M. Adeyemi Billyroset and K. Sliwa\# \\ *Hatter Institute for Cardiovascular Research in Africa: Cardiology Unit, Department of Medicine, University of Abuja Teaching Hospital, \\ Gwagwalada, Abuja. \\ \#Hatter Institute for Cardiovascular Research, Department of Medicine, Groote Schuur Hospital and the University of Cape Town, \\ South Africa \\ tDepartment of Medical Laboratory Sciences, University of Abuja Teaching Hospital, Gwagwalada, Abuja
}

Introduction: Although brain natriuretic peptide levels has been shown in our native African Hypertensive Cohort to differentiate hypertensive left ventricular hypertrophy (LVH) without heart failure (HF) from hypertensive HF due to systolic and/or diastolic dysfunction, it has not been very helpful in differentiating hypertensive subjects with LVH from those without. There is therefore the need to look for other biomarkers that can effectively do this.

Objectives: We therefore decided to study the role of the novel biomarker ST2 in differentiating the different spectrum of hypertension (hypertension without LVH from hypertensive LVH, and hypertensive LVH from hypertensive heart failure) in some of our $200 \mathrm{Nigerian}$ hypertensive cohorts.

Methods: It is a prospective cohort study echocardiography was performed on all subjects. Measurements taken include left ventricular dimensions and transmitral pulse wave Doppler flow. LVH was considered present when left ventricular interventricular septal wall thickness in diastole and/or left ventricular posterior wall thickness in diastole exceeded I 3mm. Plasma ST2 was measured using electrochemiluminescence type immunoassay. 
Results: Preliminary data on a subgroup of 40 subjects studied will be presented. $53.3 \%$ were female, $46.7 \%$ were male and mean age was $49.9 \pm 11.5$ years. There was significant difference in the ST2 levels between hypertensive subjects with LVH and those without $(49.7 \mathrm{ng} / \mathrm{ml} \pm 36.2 \mathrm{ng} / \mathrm{ml}$ versus $23.2 \mathrm{ng} / \mathrm{ml} \pm 11.7 \mathrm{ng} / \mathrm{ml}$, p-value=0.003). There was also a significant difference when the ST2 levels of those with hypertensive HF was compared with that of those with hypertensive LVH $(197.3 \mathrm{ng} / \mathrm{ml} \pm 68.1 \mathrm{ng} / \mathrm{ml}$ versus $49.6 \mathrm{ng} / \mathrm{ml} \pm 36.3 \mathrm{ng} / \mathrm{ml}$, p-value=0.000).

Conclusion: Plasma ST2 levels appear to be a very useful marker in differentiating the different spectrum of hypertension-hypertensive heart disease-hypertensive heart failure, and may hold a future role in this regard.

\title{
Persistence of escape rhythm in chronically paced patients with permanent complete heart block
}

\section{Andrzej Okreglicki and Luzaan Papenfus}

Cardiac Clinic, University of Cape Town, Groote Schuur Hospital, Observatory, South Africa

Introduction: The most recent World Society of Arrhythmias' survey published in $201 \mathrm{I}$ shows that in South Africa the implantation of pacemakers is steadily increasing by $7 \%$ annually. In Groote Schuur Hospital the majority of pacemakers are implanted for AV block (69\%). In patients with permanent complete heart block, it is the presence of an escape rhythm that determines patient survival before pacing can be implemented. It is said that a ventricular or wide complex escape rhythm is less reliable than I originating from the AV junction. With the increasing number of pacemakers, there is a greater workload on the "cathlab". At the time of pacemaker box replacement, as for battery depletion, prior knowledge of the persistence of an escape rhythm is useful because if absent, a temporary pacemaker with transvenous lead is needed to cover the patient for the short time of disconnection of the permanent pacemaker.

Aim: To determine the persistence of an underlying escape rhythm in chronically paced patients at $=3$ months after pacemaker implantation. Methods: A retrospective review of pacing and routine follow-up records of patients of the Arrhythmia Clinic at Groote Schuur Hospital determining specifically whether an escape rhythm was found when the pacemaker was either transiently turned off or slowed down.

Results: Preliminary data included only patients with pacemaker implantation in 201 I. Complete records, ECGs and pacemaker follow-up details were available in only 48 patients. $46 \%$ were males, 54\% females with ages: $69.0 \pm 10.5 y$ rs (range: 42 - 9 lyrs). All the patients had an escape rhythm before pacemaker implantation: it was AV junctional/ narrow in 31\% and wide/ventricular in $69 \%$. At follow-up $78.2 \%$ of the patients who initially presented with a narrow escape still had an escape rhythm vs $63.3 \%$ of the initial wide-complex escape group.

Conclusion: Escape rhythms do not persist chronically in all patients who initially had an escape on presentation with complete heart block whether the escape rhythm QRS-complexes were narrow or wide.

\section{Evaluation of inflammatory markers in hypertensive patients vs normotensive}

\author{
E. Paraskevopoulou*, M.Skoura", B.Geladakit, E. Kastrinelli", A. Xanthopoulos*, S.Patsilinakos" and P. Paraskevopoulou \\ "Cardiology Clinic, Konstantopouleio General Hospital of Nea lonia, Greece \\ \#Biochemistry, Konstantopouleio General Hospital of Nea lonia, Greece \\ tHematology Department, Konstantopouleio General Hospital of Nea lonia, Greece
}

Introduction: Chronic vascular inflammation plays an important role in the development of essential hypertension and may contribute to the target organs damage. Elevated levels of Matrix metalloproteinase enzymes, Myeloperoxidase of white blood cells and IL-6 may play role in arterial stiffness and endothelial dysfunction.

Aim: The aim of this study is to evaluate the significance of elevated levels of Matrix metalloproteinase enzyme ( MMP-10), Myeloperoxidase of white blood cells (MPO), Interleukine -6 (IL-6), White blood cells (WBC) and the absolute number of Neutrophils as imflammatory markers in hypertensive patients. The elevated levels of these markers may play an important role in endothelial dysfunction and target organ damage. Material and methods: 92 hypertensive patients, 36 males and 56 females (aged $66 \pm 8.4$ ) and 40 normotensive, 16 males and 24 females (aged $43.4 \pm 7$ ) were the participants of this study. All the hypertensive patients were treated with anti-hypertensive medications (ACEl, angiotensin receptor blockers, beta adrenergic blockers, calcium channel blockers alone or in a combination therapy). The plasma concentration 
of MMP-IO and IL-6 were measured in an automated immunoassay analyser (Brio of Diachel), the first with an R\&D Systems Elisa kit and the second with a Biosource Elisa kit. MPO levels were measured in plasma with an enzymatic immunoassay one stage method, Siemens reagents in Dimension EXL analyser. From a Full Blood Count measurement (ADVIA 2120 automated hematology analyser) we evaluated the number of WBC and the absolute number of Neutrophils.

Results: Plasma levels of MMP-10, MPO and IL-6 were elevated in hypertensive patients vs normotensive patients. The mean and SD of these parameters were: MMP-I0: $644 \pm 289$ vs 39I \pm 198 ( $p$-value=0.00I); MPO: $781.5 \pm 41.8$ vs |82.9 \pm |8.2 (p-value=0.027); IL-6: $65.3 \pm 22.6$ vs $33.7 \pm 17.6$ ( $p$-value=0.000); WBC: $7.6 \pm 1.6$ vs $7.4 \pm 1.7$ ( $p$-value=0.66 - NS); Neutrophils (absolute number): $4.3 \pm 1.2$ vs $4.3 \pm 1.5$ ( $p$-value=0.99 - NS). The data analysis was performed with SPSS 16.0.

Conclusion: Matrix metalloproteinases as proteolytic enzymes that degrade the extracellular matrix and basement membrane, MPO and IL-6 as important markers of inflammation have increased interest in the investigation of risk factors for cardiovascular diseases. The elevated levels of MMP- I0, MPO and IL-6 in hypertensive patients may play an important role in the damage of target organs due to hypertension.

\title{
Red blood cell distribution width (RDW) as a new biological marker of heart failure - correlation with brain natriuretic peptide (BNP)
}

\section{E. Paraskevopoulou", A. Xanthopoulos", I. Stergiou\#, E. Kastrinelli", M. Skourat, H.Theofanakist, P. Paraskevopoulou ${ }^{\dagger}$ and}

\section{S.Patsilinakos*}

"Cardiology Clinic, Konstantopouleio General Hospital of Nea lonia, Greece

\#Biochemistry, Konstantopouleio General Hospital of Nea lonia, Greece

tHematology Department, Konstantopouleio General Hospital of Nea lonia, Greece

Introduction: Recent studies show a growing interest in RDW, a simple parameter of the standard full blood count, whose elevated levels seem to have prognostic value in patients with heart failure (HF) correlating with poor prognosis and high mortality rates.

Aims: The aim of this study is to investigate the RDW levels in patients with HF (NYHA =2) and the correlation with the BNP levels

Design - method: The study included 215 patients, 142 males and 73 females, aged from 50 to 74 years which were distributed into 3 study groups: group A (n:86) consisted of patients with HF due to valvulopathies, group B (n:99) patients with ischaemic cardiomyopathy and group $\mathrm{C}$ (n:30) patients with HF, atrial fibrillation and hypertensive cardiomyopathy. BNP levels were measured in all patients and RDW were obtained as part of the complete blood count. Patients with anaemia (heterozygous b-thalassaemia, iron deficiency anaemia, folic acid or vitamin B 2 deficiency, bleeding) were excluded from this study. A total of 17 patients were excluded from this trial, 7 patients from group $A$, 6 patients from group $B$ and 4 patients from group C. RDW levels were measured in all 3 groups in correlation with the BNP levels.

Results:The correlation (Pearson coefficient) between RDW/BNP in the 3 groups of patients were: 0.476 in Group A* (n:79); 0.5 I 2 in Group B (n:93) and 0.06I in Group C(n:26). "Correlation is significant at the 0.0 l level (2-tailed).

Conclusion: The study results show significant correlation in the groups A and B between BNP and RDW but there is no correlation in group C. RDW is a readily available and inexpensive marker with prognostic value in patients with HF and it could be proved that RDW has a diagnostic value in emergency medicine. For better determination of its prognostic value future studies have been programmed including a greater number of patients and follow-up was planned for all patients on a 6 months - I year basis.

\section{Mitral regurgitation in congenital submitral aneurysm (SMA): a clinical and echocardiographic perspective}

\author{
Anupa Patel, Ferande Peters, Hiral Matioda, Elena Libhaber, Farouk Mamdoo and Mohammed R. Essop \\ Division of Cardiology, Chris Hani Baragwanath Hospital, University of the Witwatersrand, Johannesburg, South Africa
}

Introduction: Congenital submitral aneurysm (SMA) is a rare disorder which has a unique appearance, important clinical complications and is confined almost exclusively to Africans of Sub-Saharan origin. Mitral regurgitation (MR) is an important accompanying clinical feature. 
Objective: To prospectively document the prevalence and mechanisms of mitral regurgitation in SMA in the modern era of ultrasound imaging. Methods: Between January 2009 and February 201 I, 13 subjects, all of African descent were diagnosed with SMA using 2 dimensional (2D) and 3 dimensional (3D) transthoracic and transoesophageal echocardiography. Assessment of MR was performed in accordance with the American Society of Echocardiography guidelines on valvular regurgitation. 2D transthoracic echo and tissue motion annular displacement (TMAD) were used to detect systolic dyssynchrony of the mitral annulus (MAD).

Results: The mean age was 40.5 years, 8 (61.5\%) were female. Most - 10 subjects (76.9\%) - were referred for a either an incidental murmur or acute heart failure. The mean left ventricular end-diastolic diameter (LVEDD) was $55.8 \mathrm{~mm}$ and mean left ventricular ejection fraction (LVEF) was $55.4 \%$. All subjects had a single aneurysm. The most common location was along the posterior annulus - 6 subjects (46.2\%). A pan-systolic murmur, grade $2 / 6$ or more was heard in all subjects. 2 subjects (15.4\%) had moderate MR while the remaining II subjects (84.6\%) had severe MR. The leaflets appeared morphologically normal in all cases. Restricted motion or tethering of the leaflet segment adjacent to the aneurysm occurred in 7 subjects (53.9\%), pseudo-prolapse of the P2 segment occurred in 2 subjects (I5.4\%) while 4 subjects (30.8\%) had normal leaflet motion. In 2 subjects (15.4\%), rupture into the left atrium contributed to the severity of the MR. A further important contributing factor, was that in all subjects, the area of the annulus adjacent to the commissure appeared to be defective, with part of the wall of the aneurysm involving this area instead of annular tissue. In all subjects, mitral annular dilatation occurred. MAD was noted in 6 subjects (46.2\%).

Conclusion: Significant MR is common in SMA and is caused by multiple mechanisms that need to be identified systematically to guide surgical repair.

\title{
Morphologic assessment of the rheumatic mitral valve
}

\author{
Alfonso Pecoraro, Zaheera Cassimjee, Philip Herbst and Anton Doubell \\ Division of Cardiology, Department of Medicine, University of Stellenbosch and Tygerberg Hopsital, South Africa
}

Introduction: The pathophysiological hallmark of rheumatic valve disease is a valvulitis which causes commissural fusion. The sequelae of commissural fusion give rise to the morphology and physiology of rheumatic valve disease. This is neglected in the current WHO criteria for the diagnosis of subclinical rheumatic mitral valve disease. The recently proposed combined criteria (CC) aim to address this shortcoming, but ignore commissural fusion and the secondary morphologic features associated with commissural fusion. We describe the commissures in patients with rheumatic and non-rheumatic valve disease and contrast the differences in secondary morphologic features of commissural fusion. Methods: Patient selection was based on a keyword search of echocardiographic reports and prospective enrolment of the first 15 patients fulfilling criteria of each of 4 cohorts. Cohort I: Mitral Stenosis (MS); Cohort 2: Rheumatic Heart Disease (RHD) as defined by CC but without MS; Cohort 3: Dilated cardiomyopathy (DCM) with leaflet restriction due to tethering; Cohort 4: Normal controls. All patients had detailed clinical assessment and transthoracic echocardiography. The commissures were assessed both via direct inspection of the commissures and assessing secondary features of commissural fusion namely: anterior mitral valve leaflet (AMVL) diastolic bowing, AMVL short axis diastolic shape, AMVL to commissure angle during diastole and mitral valve area (planometry). These features were then compared in all cohorts.

Results: Commissural fusion was observed in all Cohort I and 2 patients $(n=30)$, with bilateral commissural fusion observed in the majority of patients (87\%). All patients with commissural fusion had AMVL diastolic bowing. No AMVL diastolic bowing was observed in the dilated cardiomyopathy or normal control groups. The vertical commissure separation distance was significantly less in Cohort 1 and 2 compared to Cohort 3 and 4 (3.46mm vs. $3.8 \mathrm{~mm}$ vs. $8.6 \mathrm{~mm}$ vs. $8.3 \mathrm{~mm}, \mathrm{p}=0.0 \mathrm{I})$. Compared to Cohorts 3 and 4 , Cohort 2 patients had significantly smaller mitral valve area $\left(5.68 \mathrm{~cm}^{2}\right.$ vs. $5.72 \mathrm{~cm}^{2}$ vs. $\left.3.36 \mathrm{~cm}^{2}, \mathrm{p}=0.0 \mathrm{I}\right)$.

Conclusion: Commissural fusion and the secondary feature of AMVL bowing during diastole are typical morphologic features of rheumatic mitral involvement. Addition of these features should improve the sensitivity and specificity of current criteria for the diagnosis of subclinical rheumatic mitral valve disease. 


\title{
HDL/Sphingosine-I-phosphate protects SAFEly: a potential role of the mitochondria in conferring cardioprotection
}

\author{
Sarah Pedretti", Damian Hacking", Miguel A. Frias", Richard W. James" and Sandrine Lecour \\ "Hatter Institute for Cardiovascular Research, Department of Medicine, Groote Schuur Hospital and the University of Cape Town, \\ South Africa \\ \#Clinical Diabetes Unit, Department of Internal Medicine, Medical Faculty, University of Geneva, Geneva, Switzerland
}

Purpose: High density lipoprotein cholesterol (HDL) and its component sphingosine-I-phosphate (SIP) protect against myocardial infarction. Recently the SAFE (survivor activating factor enhancement) pathway involving tumour necrosis factor (TNF) and the transcription factor signal transducer and activator of transcription 3 (STAT-3), has been identified as a key signalling pathway in cardioprotection, although the end effector remains unclear. Opening of the mitochondrial Permeability Transition Pore (mPTP) is a crucial initiator of cell death in the setting of myocardial infarction. We therefore hypothesised that HDL/SIP protect the heart via the activation of the SAFE pathway and the subsequent inhibition of mPTP opening.

Methods: Isolated adult cardiomyocytes from wildtype, STAT-3 cardiomyocyte deficient (STAT3 -/-) or TNF deficient (TNF -/-) mice were

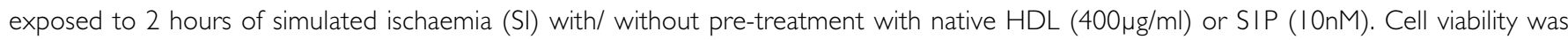
assessed using trypan blue staining and mPTP opening was measured using TMRM fluorescence and normalised to the normoxic control.

Results: Cardiomyocyte viability under normoxic conditions was $76.2 \pm 2.6 \%$. SI reduced viability to $56.8 \pm 3.2 \%$ ( $p<0.0$ I vs. control). Cardiomyocyte viability was restored with SIP $(74.9 \pm 1.6 \%)$ or native HDL $(71.2 \pm 2.8 \%)(p<0.00$ I vs. SI). TMRM fluorescence following SI was $71.3 \pm 10.5 \%(p<0.000$ I vs. control), and restored to $96.0 \pm 2.3 \%$ in the SIP group and $79.2 \pm 3.6 \%$ in the HDL group ( $<<0.05$ vs. SI). In the STAT3 -/- and TNF -/- mice, no cardiomyocyte viability improvement or restoration of TMRM fluorescence was observed with either HDL or SIP.

Conclusion: Both native HDL and SIP are cardioprotective and our data strongly suggest that this effect is mediated via activation of TNF, STAT-3 and subsequent inhibition of mPTP opening.

\section{GST polymorphisms and the risk of early onset coronary artery disease in young South African Indians}

\author{
Alisa Phulukdaree*, Sajidah Khan", Devapregasen Moodley and Anil Chuturgoon* \\ "Discipline of Medical Biochemistry, School of Laboratory Medicine and Medical Science, Faculty of Health Science, \\ University of KwaZulu-Natal, Durban, South Africa \\ \#Department of Cardiology, University of KwaZulu-Natal, Durban, South Africa \\ tDepartment of Microbiology and Immunobiology, Harvard Medical School, Boston, Massachusetts, United States of America
}

Background: Glutathione-S-transferases (GSTs) detoxify environmental agents which may influence the onset and progression of disease. Dysfunctional detoxification enzymes are responsible for prolonged exposure to reactive molecules and can contribute to endothelial damage. Endothelial damage is one of the underlying factors in the development of coronary artery disease (CAD). Epidemiological data in SA show Indians have the highest mortality rates for CAD followed by mixed race, white and black Africans. Literature shows a strong familial link with the history of diabetes mellitus type 2, hypertension and CAD, supporting a genetic basis for the development of the disease.

Aim: This study aimed to assess two common polymorphic variants in GST (GSTPI and GSTMI), in young patients with CAD.

Methods: All patients $(n=100)$ were South Africans with Indian ancestry. A corresponding age, sex and race matched control group $(n=102)$ was also recruited. The frequency of the GSTPI AI05/GI05 and GSTMI +/0 alleles was assessed by PCR-RFLP and differential PCR, respectively.

Results: The GSTPI AI05/AI05 and GSTMI 0/0 genotype occurred at a higher frequency in CAD patients (65\% vs. 48\% and 36\% vs. 18\%, respectively). A significant association between GSTPI AI05 allele (OR=0.60II; 95\% Cl=0.3803-0.9503; $p=0.0377)$ and GSTMI 0/0 $(O R=2.593 ; 95 \% \mathrm{Cl}=1.353-4.971 ; p=0.0043)$ genotype in CAD was observed. Furthermore, we found a significant association between smoking and CAD, and the presence of the respective genotypes together with smoking increases the risk for CAD (GSTPI AI05 RR=I.382; $95 \% \mathrm{Cl}=0.958-1.994 ; p=0.0987$ and GSTMI null $R R=1.725 ; 95 \% \mathrm{Cl}=1.044-2.85 \mathrm{I} ; \mathrm{p}=0.022 \mathrm{I}$ ). 
Conclusion: The high percentage of patients who smoked and presented with the GSTPI AI05/AI05 and GSTMI 0/0 genotypes supports the theory that GSTs play an important role in CAD. These results support the association of GSTMI null genotype, the GSTPI AI05 allelotype and smoking with CAD.

\section{The interaction of common polymorphisms of the lipoprotein lipase and PONI genes with cardiovascular risk factors in the Phoenix community}

\section{Rosaley Prakaschandra*, Michelle Gordon" and D.P. Naidoo"}

"Department of Cardiology, Inkosi Albert Luthuli Central Hospital and University of KwaZulu-Natal, Durban, South Africa

\#Department of Cardiology, Division of Medicine, University of Kwazulu-Natal, Durban, South Africa

Background: We investigated gene polymorphisms related to oxidative stress and dyslipidaemia in a sample of subjects with and without metabolic syndrome (MS) from the Phoenix Lifestyle Project. Single nucleotide polymorphisms (SNP) in the Human Paraoxonase /PONI (Leu55Met and GIn192Arg SNPs) and LPL genes (N29IS and S447 X SNPs) were studied to investigate the associations of these mutations with cardiovascular risk factors and risk factor clustering in this sample.

Method: There were I 419 subjects from the Phoenix Lifestyle Project cohort who were studied for CV risk factor prevalence and clustering. Anthropometric measurements were performed, with fasting venous blood samples taken from each subject for glucose, lipid and genetic analysis. Genotyping was performed using real-time PCR, allele-specific probes and Melting Curve analysis in a sample of 916 subjects.

Results: The crude prevalence of MS in this sub-cohort, classified according to the IDF criteria was 50\% (456/9/6). The LPL and PON I genotypes were similarly distributed $(p=n / s)$ in subjects with and without MS. There were no subjects observed with the LPL N29IS mutation. There was a significant interaction between the Gln 192Arg genotype and elevated triglyceride $(>1.7 \mathrm{mmol} / \mathrm{I})$ levels $(p=0.016$; OR $=1.356)$, while overweight females $\left(B M I>23 \mathrm{~kg} / \mathrm{m}^{2}\right)$ tended to have the $L 55 \mathrm{M}$ mutation $(p=0.027 ; O R=1.91 \mathrm{I})$ which was identified in $23 \%$ of overweight females.

Discussion: Although the S447X, L55M and Q192R polymorphisms are common in this population, only the 192Arg and the 55Leu alleles, previously associated with reduced protection against lipid peroxidation, predispose to hypertriglyceridaemia in the population and general obesity in females. Gene polymorphisms related to the MS risk factors may explain a high predisposition to premature artherosclerosis.

\section{The proper delivery pressure for cardioplegic solution in neonatal cardiac surgery: an investigation of biomechanical and structural properties of neonatal coronary arteries}

\section{Normunds Sikora, Aris Lacis, Vladimir Kasyanov, Elina Ligere, Valts Ozolins and Lauris Smits}

Clinic for Paediatric Cardiology and Cardiac Surgery, Children's University Hospital, Riga, Latvia

Introduction: One of the most important issues in paediatric cardiac surgery is myocardial protection, when the cardioplegic solution mixed with oxygenated blood is injected into the coronary arteries via a pump. The general principles underlying the efficacy of cold chemical cardioplegic solutions are well established. To maintain an adequate delivery of cardioplegia, the right delivery pressure should be used. Neonates have many structural, functional, biomechanic and metabolic differences from adults. It is therefore crucial to administer the cardioplegic solution with an adequate amount of delivery pressure. If the pressure is too low or too high, it can lead to severe damage of the myocardium.

Methods: To assess the right delivery pressure of the cardioplegic solution, biomechanical properties of eleven specimens of coronary arteries from neonates $12.3 \pm 13.7$ days old and weight $4.1 \pm 0.9 \mathrm{~kg}$, retrieved at the autopsies, were investigated and compared with adult arteries. Specimens were pressurised from 0 to $200 \mathrm{mmHg}$ with the step of $20 \mathrm{mmHg}$, while maintaining the length of the sample in situ. Structural damages were investigated afterwards.

Results: There was a rapid increase of strain until the inner pressure reaches $80-100 \mathrm{mmHg}$ and not as rapid regarding to the stress in the arterial wall. When the internal pressure exceeds $100 \mathrm{mmHg}$, the strain of the arterial wall increases much slower but at the same time the wall 
stress and modulus of elasticity begin to increase rapidly. It means that the structural elements of the arterial wall have been straightened and possible damage in the wall of coronary arteries of neonates may appear. These results were compared with the biomechanical properties of arterial wall in adults and several differences have been found. Structural investigations show damage to the neonatal coronary artery wall after it was being pressurised with an inner pressure $>100 \mathrm{mmHg}$ compared to the coronary artery of adult.

Conclusion: Our experimental results show that the delivery pressure of the cardioplegic solution in neonatal coronary arteries should not exceed $100 \mathrm{mmHg}$. Higher pressure increases the risk of structural damage to the vascular wall, leading to injury of the myocardium.

\section{Proteomic characterisation of cardiac endothelial cell responses to TNF-Alpha and asymmetric dimethylarginine (ADMA) stimulation}

\section{Hans Strijdom, Salome Smit, Corli Westcott, Mashudu Mudau and Amanda Genis}

Division of Medical Physiology, Department of Biomedical Sciences, Faculty of Health Sciences, University of Stellenbosch, South Africa

Introduction: Harmful stimulation of endothelial cells by TNF-alpha (pro-inflammatory cytokine) and ADMA (competitive eNOS antagonist) is observed in cardiovascular risk factor conditions.

Aim: In this study, we aimed to characterize proteomic responses (mass spectrometry analysis) and selected protein pathways (Western blotting) in cardiac endothelial cells subjected to TNF-alpha (5ng/ml; 24h) and ADMA (500uM; 24h) stimulation.

Method: TNF-alpha and ADMA stimulation resulted in the detection of 141 and 93 newly expressed proteins respectively. Protein expression induced by TNF-alpha was associated with increased regulation of cell death, apoptotic mitochondrial changes, and Ras signalling. Conversely, TNF-alpha resulted in the loss of the insulin signalling pathway, cell proliferation, calcium-binding, membrane rafts, and endosome function. Measurements of eNOS - NO signalling revealed a significant reduction in phosphorylated eNOS / total eNOS ratio $(p<0.05)$; however, no changes in $\mathrm{NO}$-production were observed as a consequence. P22-phox expression increased by 25\% ( $\mathrm{P}<0.05)$, suggestive of $\mathrm{NADPH}$-oxidase activation. TNF-alpha-induced expression of NF-KB and TRADD proteins, and a reduction in IKB-alpha expression $(27 \%$; $p<0.05)$ suggested TNFRI-TRADD-NFKB activation. Membrane integrity remained intact (propidium iodide staining). ADMA-stimulation resulted in increased ribosomal activity and protein translation, increased expression of apoptotic pathways, increased redox activity, increased cytoskeletal and vesicular activity, and increased ATPase function. Conversely, ADMA resulted in the loss of cell morphogenesis and NO synthase binding. The phosphorylated/total eNOS ratio was not affected by ADMA, and neither was NO production. NADPH-oxidase activity (p22-phox) was also not affected by ADMA, and the NF-KB pathway was not activated (IKB-alpha expression). Despite upregulation of pro-apoptotic protein pathways, cell death did not occur (propidium iodide staining).

Conclusion: The proteome of TNF-alpha stimulated cells showed significant increases in pro-apoptotic protein pathways and pro-oxidative stress protein expression, probably mediated via the TNFRI-TRADD-NF-KB pathway. Protective cellular components such as insulin signalling and eNOS activation were down-regulated by TNF, although these changes did not result in cell death. Interestingly, our data showed increases in protein synthesis, cytoskeletal and vesicular transport mechanisms in ADMA-treated cells, unrelated to its more famous role as an eNOS antagonist. Although apoptotic proteins were increased by ADMA, this was not as pronounced as in TNF-alpha-treated cells. This study provides a comprehensive characterisation of cardiac endothelial cell proteomic responses to TNF-alpha and ADMA and novel insights into the expression of new proteins induced by these stimuli.

\section{Novel sarcomeric hypertrophy modifier proteins that interact with titin in hypertrophic cardiomyopathy}

\section{Carol Todd, Jacoba M. Mouton, Johanna C. Moolman-Smook and Craig J. Kinnear}

University of Stellenbosch Medical Research Centre (US/MRC), Centre for Molecular and Cellular Biology, Department of Biomedical Sciences, University of Stellenbosch, South Africa

Introduction: Hypertrophic cardiomyopathy (HCM) is an autosomal dominantly inherited cardiac disorder characterised by left ventricular hypertrophy $(\mathrm{LVH})$ and increased risk of sudden cardiac death. LVH is an important predictor of morbidity and mortality and is a feature of 
complex disorders such as hypertension and diabetes. Since LVH is a primary feature of HCM, this disorder has been considered a model disease to study the complex mechanisms leading to LVH. More than I $000 \mathrm{HCM}$ causing mutations in 19 genes have been identified to date. Most causal mutations have been identified in sarcomeric and myofilament associated genes. Interestingly, a large degree of phenotypic variability exists across families as well as within families harbouring the same primary HCM-causing mutation. This observation has directed the focus of several research endeavours to the identification of possible genetic modifiers of the hypertrophic phenotype.

Aim: Here, we aim to identify putative HCM-modifying genes by searching for novel interactors of Titin, a protein known to be involved in HCM. We hypothesise that proteins that interact with proteins known to be mutated in HCM can be considered potential HCM-causing or modifying genes.

Method: To identify novel Titin-interacting proteins, yeast two-hybrid analysis was performed using the II domain super repeat of Titin, found in the C-zone, as "bait" because this region of the sarcomere contains several HCM causative genes. 27 possible putative Titin interactors were identified and five were selected to be verified further. The putative interactions were confirmed by three-dimensional colocalisation. The most promising results are between Titin and Heart-type fatty acid-binding protein 3 (H-FABP3), Phosphatidylethanolamine-binding protein 4 (PEBP4) and Filamin C (FLNC). The two other interactions were between Titin and Myomesin I (MYOMI) and Myomesin 2 (MYOM2). The genes encoding these interactors will be used in family-based association studies to determine whether they are potential HCMmodifying genes.

Conclusion: The identification of HCM-modifying genes may help researchers better understand the molecular mechanisms involved in the development of LVH in HCM as well as in hypertension and diabetes. They will allow for the improved understanding of HCM patho-aetiology and may improve risk profiling.

\title{
Baroreceptor sensitivity, cardiovascular responses and ECG left ventricular hypertrophy in men: the SABPA study
}

\author{
Lisa Uys*, Leoné Malan*, Johannes van Rooyen*, Faans Steyn\#, Tjalf Ziemssent and Manja Reiman' \\ "School for Physiology, Nutrition and Consumer Sciences, North-West University, Potchefstroom Campus, Potchefstroom, South Africa \\ \#Statistical Consultation Service, North-West University, Potchefstroom Campus, Potchefstroom, South Africa \\ tDepartment of Neurology, Medical Faculty Carl Gustav Carus, Dresden University of Technology, Germany
}

Introduction: Research has established a significant relationship between hypertension and attenuated baroreceptor sensitivity (BRS), which in turn indicates an alteration in autonomic control of the cardiovascular system. Sympathetic hyperactivity has been implicated as one of the possible contributing factors to hypertension specifically in black African men. The objective of this study was to compare BRS and cardiovascular responses between African and Caucasian men. Associations between BRS, cardiovascular responses, ambulatory blood pressure and left ventricular hypertrophy (LVH) were also investigated in these groups.

Methods: The Sympathetic Activity and Ambulatory Blood Pressure in Africans study (SABPA) included 82 African and 100 Caucasian male teachers (aged 20 - 65 years), recruited in the North-West Province, South Africa. Ambulatory blood pressure measurements were conducted which was followed the next morning by cardiovascular measurements registered with a Finometer and I2-lead ECG during rest as well as stressor exposure (Stroop colour-word conflict and cold pressure test). Spontaneous BRS and Cornell product (marker of LVH) were calculated. From the 12-lead ECG, the height of the R-wave in the aVL lead was also observed as marker for increased cardiovascular risk.

Results: The African men had significantly higher blood pressure $(p<0.001)$ and Cornell product $(p=0.026)$ as well as lower BRS stress responses $\left(p=0.012\right.$ ). In this group, attenuated $B R S$ (Adjusted $R^{2}=0.59 ; \beta=-0.24 ; 95 \% C l=-0.42 ;-0.06$ ), coupled to an $\alpha$-adrenergic response pattern (Adjusted $\mathrm{R}^{2}=0.58$; $\beta=-0.84 ; 95 \% \mathrm{Cl}=-1.08$; -0.60 ) was associated with elevation of ambulatory blood pressure. $\mathrm{LVH}$ was, however, not predicted by a BRS reduction.

Conclusion: Attenuated BRS, especially during stress is a possible contributing factor to the development or promotion of $\alpha$-adrenergic driven hypertension in our male African group. Coupled to higher RaVL and Cornell product values, the risk for future cardiovascular events may be increased. 


\title{
Cardiac presentations of HIV positive patients at Charlotte Maxeke Johannesburg Academic Hospital
}

\author{
Ahmed Ismail Vachiat, Riaz Garda, Lance Mkhwanzi, Keir McCutcheon, Thomas Kalk, Rohan Ramjee and Pravin Manga \\ Division of Cardiology, Charlotte Maxeke Johannesburg Academic Hospital, University of the Witwatersrand, Johannesburg, South Africa
}

Introduction: South Africa has over 5.6 million people infected with HIV. There is limited knowledge on the impact of the HIV epidemic on heart disease in South Africa. Thus the aim of this study was to ascertain the spectrum of cardiac disease in HIV positive patients presenting to a busy public academic cardiology unit.

Methods: HIV positive patients presenting to the Cardiology department at CMJAH over 2 years (20I0 and 20II) were reviewed. Data was collected with regard to spectrum of cardiac disease, the CD4 count and status of anti-retroviral therapy.

Results: There were 204 HIV positive patients out of 2500 admissions (8.2\%) that presented to the Cardiology unit. The average age was 42 years with 89 (43.6\%) males and I 5 (56.4\%) females. The most common condition was HIV cardiomyopathy 53 (26\%) patients. There were 39 (19\%) patients that presented with valvular heart disease and 34 (18\%) patients that presented with pericardial effusions. 23 (I 4\%) patients presented with coronary artery disease. I I patients presented with STEMI and unstable angina and 5 with NSTEMI. 16 (8\%) patients presented with right heart failure, 10 (5\%) patients with hypertensive cardiomyopathy, 6 (3\%) with constrictive pericarditis, 7 (4\%) with peripartum cardiomyopathy, 3 (2\%) with infective endocarditis and 7 (3\%) with other causes of cardiovascular disease. There were 18 (9\%) hypertensive patients and $9(5 \%)$ diabetic patients. 57 patients (28\%) were diagnosed with Tuberculosis. Only 41 (20\%) patients were on anti-retroviral therapy on admission. $75 \%$ of patients had CD4 count $<350$ cells/ $\mu$ l. There were 5 (3\%) patients with malignancies. Hypertrophic cardiomyopathy was diagnosed in 4 patients, dextrocardia in I patient and left ventricular non-compaction in I patient. The majority of patients were in sinus rhythm except 3 patients with atrial fibrillation and I patient with atrial flutter. One patient presented with Torsades du Pointes.

Conclusion: HIV positive patients present at a young age and are more likely to be female. HIV cardiomyopathy, valvular heart disease and pericardial effusions are the most common presentations. This study showed an almost 5-fold higher prevalence of CAD as compared to a previous review in 2006 - 2008. The majority (80\%) of patients were not on anti-retroviral therapy although $87 \%$ of these patients qualified for anti-retroviral therapy.

\section{Myocardial twist characteristics in an African chronic kidney disease population on haemodialysis before and after dialysis as measured by speckle tracking echocardiography}

\author{
Anthony Yip*, Saraladevi Naicker\#, Ferande Peters", Mduduzi Mashabane”, Elena Libhaber*, Nirthi Maharaj*, \\ Samantha Govender*, Hiral Matioda* and Mohammed R. Essop* \\ "Division of Cardiology, Chris Hani Baragwanath Hospital, University of the Witwatersrand, Johannesburg, South Africa \\ \#Department. of Medicine and Division of Nephrology, Charlotte Maxeke Johannesburg Academic Hospital, University of the Witwatersrand, \\ Johannesburg, South Africa
}

Introduction: Haemodialysis causes variable loading conditions on the heart affecting measurement of ventricular function by ejection fraction. Myocardial twist, defined as the 'wringing' action of the heart, describes an aspect of myocardial deformation which results in clockwise rotation at the base of the heart, and counter-clockwise rotation at the apex. Net twist is the difference between peak apical and basal rotation, and represents a novel way to assess myocardial function. In this pilot study, the net twist was measured in an African population before and after haemodialysis compared to matched controls.

Methods: Twenty one patients were recruited from the $\mathrm{CH}$ Baragwanath Hospital haemodialysis unit. Transthoracic echocardiography was performed before and after a single dialysis session using an IE33 system with offline speckle tracking and myocardial twist measurements made using the Excelera system (Philips Ltd). Twenty two age and gender matched controls were similarly echoed.

Results: The mean age of the control vs. renal group was 43.77 years and 43.95 years (male: $43 \%$ and $41 \%$ respectively). Apical rotation was significantly diminished in renal patients compared to their controls $\left(5.25^{\circ} \mathrm{vs} .6 .29^{\circ}, p=0.04\right)$, but did not appear to be affected by load changes after a single dialysis session ( $5.25^{\circ}$ vs. $5.89^{\circ}, p=0.12$ ). In comparison, no statistical difference was seen in basal rotation and net twist characteristics between renal patients against the control group, nor after dialysis.

Conclusion: Apical rotation is diminished in chronic kidney disease on haemodialysis compared to matched controls, but does not appear to be significantly affected by the load changes of dialysis. 